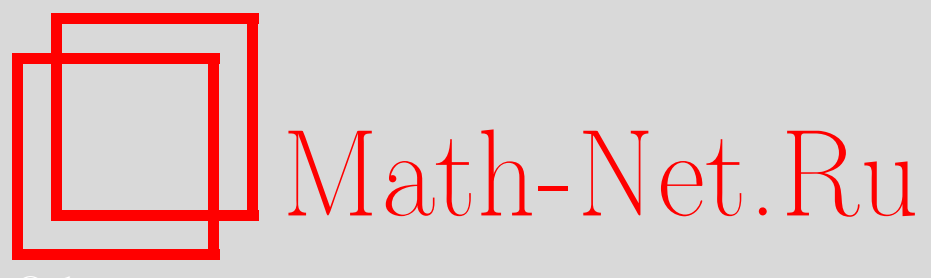

А. М. Вершик, П. Б. Затицкий, Ф. В. Петров, Виртуальная непрерывность измеримых функций многих переменных и ее приложения, УМН, 2014, том 69, выпуск $6,81-114$

DOI: https://doi.org/10.4213/rm9628

Использование Общероссийского математического портала Math-Net.Ru подразумевает, что вы прочитали и согласны с пользовательским соглашением http://www . mathnet.ru/rus/agreement

Параметры загрузки:

IP: 54.80 .73 .141

26 апреля 2023 г., 13:58:08

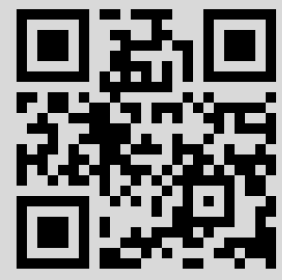




\title{
Виртуальная непрерывность измеримых функций многих переменных и ее приложения
}

\author{
А. М. Вершик, П. Б. Затицкий, Ф.В. Петров
}

Классическая теорема Лузина утверждает, что измеримая функция одной переменной "почти" непрерывна. Для измеримых функций нескольких переменных аналогичное утверждение (непрерывность на произведении множеств почти полной меры) уже не всегда имеет место. Поиск правильного аналога этой теоремы приводит к понятию виртуально непрерывных функций нескольких переменных. Это, по-видимому, новое понятие неявно присутствует в утверждениях типа теорем вложения и теорем о следах для пространств Соболева и фактически вскрывает их природу как теорем о виртуальной непрерывности. Из наших результатов следует, что в условиях теорем Соболева интегрирование функции возможно по очень широкому классу сингулярных мер, включая как частный случай меры, сосредоточенные на подмногообразиях. Понятие виртуальной непрерывности используется и для классификации измеримых функций нескольких переменных, а также в ряде вопросов теории динамических систем, теории полиморфизмов и бистохастических мер. В этой работе мы напоминаем необходимые определение и свойства допустимых метрик, приводим ряд определений виртуальной непрерывности и обсуждаем некоторые приложения. Сокращенная версия (без доказательств) опубликована в [22].

Библиография: 24 названия.

Ключевые слова: допустимые метрики, виртуальная топология, теоремы о следах, бистохастические меры, теоремы вложения.

DOI: $10.4213 / \mathrm{rm} 9628$

\section{СОДЕРЖАНИЕ}

1. Введение. Допустимые метрики ............................ 82

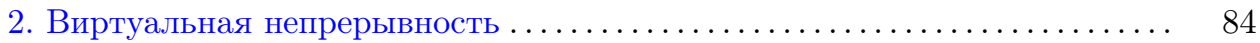

2.1. Теорема Лузина об измеримых функциях одного аргумента .... 84

2.2. Определение виртуальной непрерывности и первые примеры ... 85

В основу статьи положен доклад первого автора на конференции "Современные проблемы математики, механики и математической физики”, посвященной 150-летию со дня рождения В. А. Стеклова.

Работа выполнена при поддержке РФФИ (гранты № 14-01-00373-а и 13-01-12422-офи-м), гранта Президента РФ МК-6133.2013.1, Исследовательской лаборатории им. П. Л. Чебышёва СПбГУ (грант Правительства РФ, дог. № 11.G34.31.0026), ОАО "Газпром нефть" и СПбГУ (грант № 6.38.223.2014). 
2.3. Дальнейшие свойства виртуально непрерывных функций .. 86

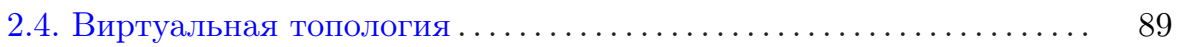

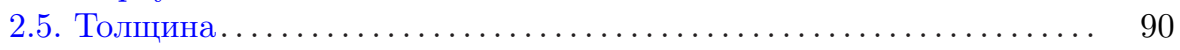

2.6. Бистохастические меры и полиморфизмы ............... 98

2.7. Норма в пространстве виртуально непрерывных функций . . . . 100

3. Приложения: транспортная задача, теоремы вложения, теоремы о сле-

дах и ограничение метрик ......................... 107

3.1. Двойственность Канторовича в транспортной задаче........... 107

3.2. Пространства Соболева и теоремы о следах ................. 111

3.3. Ядерные операторы в гильбертовом пространстве............ 111

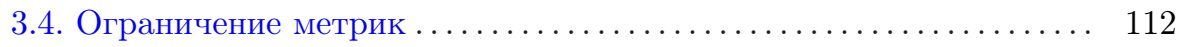

Список литературы . . . . . . . . . . . . . . . . . . . . . . . . 113

\section{1. Введение. Допустимые метрики}

Мы рассматриваем стандартное вероятностное пространство с непрерывной (т. е. неатомической) мерой (пространство Лебега-Рохлина), которое изоморфно отрезку [0, 1] с лебеговой мерой. В работах [13], [16], [19] первого автора предлагалось рассматривать на фиксированном стандартном пространстве с мерой $(X, \mathfrak{A}, \mu)$ различные (допустимъє) метрики - в противоположность обычному подходу, при котором фиксируется, наоборот, метрическое пространство и рассматриваются различные борелевские меры на нем. Новый подход полезен и необходим в эргодической теории и в других ситуациях. Согласование структур измеримого и метрического пространств приводит к понятию метрической (или допустимой) тройки.

ОПРЕДЕлЕниЕ 1. Метрика (или полуметрика) $\rho$, измеримая как функция двух переменных на стандартном пространстве с мерой $(X, \mathfrak{A}, \mu)$, называется допустимой, если существует измеримое подмножество $X_{0} \subset X$ полной меры, $\mu\left(X_{0}\right)=1$, такое, что метрическое (соответственно полуметрическое) пространство $\left(X_{0}, \rho\right)$ сепарабельно.

Стандартное пространство с мерой $(X, \mu)$, наделенное допустимой (полу)метрикой $\rho$, называется допустимой метрической тройкой или просто допустимой тройкой $(X, \mu, \rho)$.

Свойства допустимых полуметрик и метрик подробно изучены в работах [10], [24]. В частности, там приведен ряд равносильных определений этого понятия.

Пользуясь стандартностью пространства, несложно установить следующее предложение.

ПреДЛОжЕНИЕ 1. Если $\rho$ - допустимая метрика на $(X, \mathfrak{A}, \mu)$, то пополненная борелевская сигма-алгебра $\mathfrak{B}=\mathfrak{B}(X, \rho)$ совпадает с сигма-алгеброй $\mathfrak{A}$, мера $\mu$ при этом внутренне регулярна относительно метрики $\rho$, т.е. для любого $A \in \mathfrak{A}$ имеется равенство

$$
\mu(A)=\sup \{\mu(K): K \subset A, K \text { компактно в метрике } \rho\} .
$$

Тем самым, для любой допустимой метрики исходная мера $\mu$ будет мерой Радона в пространстве $(X, \rho)$. 
ДокАЗАТЕЛЬСТво. Измеримость борелевских множеств, т. е. включение $\mathfrak{B} \subset \mathfrak{A}$, была доказана в [24]. Докажем теперь внутреннюю регулярность, из которой следует обратное включение $\mathfrak{A} \subset \mathfrak{B}$. Существует некоторое подмножество $X_{0} \subset X, \mu\left(X_{0}\right)=1$, такое, что метрическое пространство $\left(X_{0}, \rho\right)$ сепарабельно. Сужение сигма-алгебры $\mathfrak{A}$ на множество $X_{0}$ обозначим $\mathfrak{A}_{0}$. Отметим, что мы можем выбрать $X_{0}$ замкнутым в $X$ по метрике $\rho$, получив таким образом $X \backslash X_{0} \in \mathfrak{B}$. Пусть $X_{1}$ - пополнение $X_{0}$ по метрике $\rho$. Зададим меру $\mu_{1}$ на борелевской сигма-алгебре $\widetilde{\mathfrak{B}}_{1}=\mathfrak{B}\left(X_{1}, \rho\right)$ польского пространства $\left(X_{1}, \rho\right)$, продолжив меру $\mu$ с $\mathfrak{B}_{0}=\mathfrak{B}\left(X_{0}, \rho\right)$ и положив $\mu_{1}\left(X_{1} \backslash X_{0}\right)=0$. Пусть $\mathfrak{B}_{1}$ пополнение сигма-алгебры $\widetilde{\mathfrak{B}}_{1}$ по мере $\mu_{1}$. Отметим, что $\left(X_{1}, \mathfrak{B}_{1}, \mu_{1}\right)$ является пространством Лебега, так как это польское пространство с борелевской вероятностной мерой на пополненной борелевской сигма-алгебре. Кроме того, отображение

$$
\text { id }:\left(X_{0}, \mathfrak{A}_{0}, \mu\right) \rightarrow\left(X_{1}, \mathfrak{B}_{1}, \mu_{1}\right)
$$

есть инъективное сохраняющее меру отображение пространств Лебега. По лемме из $[11 ;$ п. 5] при этом отображении образ любого измеримого множества также измерим. Таким образом, мы имеем включение $\mathfrak{A}_{0} \subset \mathfrak{B}_{1}$. Так как $\mathfrak{A}_{0}$ является сигма-алгеброй на $X_{0}$, сужения сигма-алгебр $\mathfrak{B}$ и $\mathfrak{B}_{1}$ на $X_{0}$ совпадают и $X_{0} \in \mathfrak{B}$, то $\mathfrak{A}_{0} \subset \mathfrak{B}$. Поскольку $\mu\left(X \backslash X_{0}\right)=0$, мы получаем $\mathfrak{A}=\mathfrak{B}$. Напомним, что любая вероятностная борелевская мера на польском пространстве внутренне регулярна. Докажем внутреннюю регулярность меры $\mu$ на (возможно, неполном) метрическом пространстве $(X, \rho)$. Рассмотрим любое множество $A \in \mathfrak{A}$. Тогда $A \cap X_{0} \in \mathfrak{B}_{1}$, и из внутренней регулярности меры $\mu_{1}$ на польском пространстве $\left(X_{1}, \rho\right)$ следует, что найдется компакт $K \subset A \cap X_{0}$ такой, что

$$
\mu(K)=\mu_{1}(K)>\mu_{1}\left(A \cap X_{0}\right)-\varepsilon=\mu(A)-\varepsilon,
$$

что и требовалось. Предложение доказано.

М. Громов в книге [3] привлек внимание к рассмотрению произвольных метрических троек $(X, \mu, \rho)$, назвав их $m m$-пространствами, и к их классификации, имея в виду классические ситуации (римановы многообразия и др.). Естественно рассматривать в этом контексте допустимые тройки. Определим эквивалентность допустимых троек как эквивалентность с точностью до обратимых измеримых изометрий, сохраняющих меру: $(X, \mu, \rho) \sim\left(X^{\prime}, \mu^{\prime}, \rho^{\prime}\right)$, если

$$
\exists T: X \rightarrow X^{\prime}, \quad T \mu=\mu^{\prime}, \quad T^{-1} \mu^{\prime}=\mu, \quad \rho^{\prime}(T x, T y)=\rho(x, y) .
$$

Вот основной результат, описывающий эту эквивалентность.

Теорема 1 (М. Громов [3], А. М. Вершик [13]). Рассмотрим отображение $F_{\rho}: X^{\infty} \times X^{\infty} \rightarrow M_{\infty}(\mathbb{R})$, заданное формулой

$$
F_{\rho}\left(\left\{x_{i}, y_{j}\right\}_{(i, j) \in \mathbb{N} \times \mathbb{N}}\right)=\left\{\rho\left(x_{i}, y_{j}\right)\right\}_{(i, j) \in \mathbb{N} \times \mathbb{N}},
$$

и снабдим бесконечное произведение $X^{\infty} \times X^{\infty}$ продакт-мерой $\mu^{\infty} \times \mu^{\infty}$. Тогда образ относительно отображения $F_{\rho}$ мерь $\mu^{\infty} \times \mu^{\infty}$, m.е. мера $D_{\rho}$ на пространстве матрич (иначе говоря, случайная матрица расстояний), называемая матричным распределением метрики $\rho$, есть полный инвариант эквивалентности метрик на пространстве с мерой. 
Таким образом,

$$
(X, \mu, \rho) \sim\left(X^{\prime}, \mu^{\prime}, \rho^{\prime}\right) \Leftrightarrow D_{\rho}=D_{\rho^{\prime}} .
$$

В работе [14] этот результат распространен на так называемые чистые измеримые функции нескольких аргументов.

В теории допустимых метрик полезна следующая лемма.

Лемма 1. Пусть $\rho_{1}, \rho_{2}$ - допустимые полуметрики на пространстве Лебега $(X, \mu)$, причем $\rho_{1}$ - метрика. Тогда для любого $\varepsilon>0$ существует измеримое множество $K \subset X$ такое, что $\mu(K)>1-\varepsilon$ и полуметрика $\rho_{2}$ непрерывна на $K \times K$ по метрике $\rho_{1}$ как функция двух переменных.

ДокАЗАТЕЛЬство. Рассмотрим допустимую метрику $\rho=\rho_{1}+\rho_{2}$. Выберем компакт $K$ в этой метрике таким образом, что $\mu(K)>1-\varepsilon$. Покажем, что $\rho$ (а тогда и $\rho_{2}$ ) как функция на $K \times K$ непрерывна по метрике $\rho_{1} \times \rho_{1}$. В силу неравенства треугольника нам достаточно доказать, что для любого $\delta>0$ найдется $\sigma>0$ такое, что если $x, y \in K$ и $\rho_{1}(x, y)<\sigma$, то $\rho(x, y)<\delta$. Если это не так, то найдутся $\delta>0$ и последовательности $\left\{x_{n}\right\},\left\{y_{n}\right\}$ точек из $K$ такие, что $\rho\left(x_{n}, y_{n}\right) \geqslant \delta$, но $\rho_{1}\left(x_{n}, y_{n}\right) \rightarrow 0$. Так как $K-$ компакт в метрике $\rho$, мы можем считать, не умаляя общности, что существуют $x, y \in K$ такие, что

$$
\rho\left(x_{n}, x\right) \rightarrow 0, \quad \rho\left(y_{n}, y\right) \rightarrow 0 .
$$

Но тогда

$$
\rho_{1}\left(x_{n}, x\right) \leqslant \rho\left(x_{n}, x\right) \rightarrow 0, \quad \rho_{1}\left(y_{n}, y\right) \leqslant \rho\left(y_{n}, y\right) \rightarrow 0 .
$$

Таким образом, мы получаем $\rho_{1}(x, y)=0$, в то время как $\rho(x, y) \geqslant \delta$. Это противоречит тому, что $\rho_{1}$ - метрика. Лемма доказана.

Из леммы 1 сразу вытекает следующее утверждение.

СлЕДСТвиЕ 1. Пусть $\rho_{1}$ и $\rho_{2}$ - две допустимые метрики на пространстве Лебега $(X, \mu)$. Тогда для любого $\varepsilon>0$ существует множество $K \subset X$ такое, что $\mu(K)>1-\varepsilon$ и топологии, задаваемье метриками $\rho_{1}$ и $\rho_{2}$ на $K$, совпадают.

\section{2. Виртуальная непрерывность}

2.1. Теорема Лузина об измеримых функциях одного аргумента. В дальнейшем мы будем говорить об (измеримых) вещественнозначных функциях, хотя большинство результатов остаются верными для отображений в стандартное борелевское пространство, в частности - в произвольное польское пространство. Общеизвестны классические теоремы Егорова и Лузина об измеримых функциях одного аргумента. Сформулируем обобщенную теорему Лузина для произвольной допустимой тройки.

СледствиЕ 2 (теорема Лузина). Пусть $\rho$ - допустимая метрика на пространстве Лебега $(X, \mu)$, a $f$ - измеримое отображение из $X$ в польское пространство $(M, d)$. Тогда для любого $\varepsilon>0$ существует множество $K \subset X$ такое, что $\mu(K)>1-\varepsilon$ и функиия $f$ на $K$ непрерывна по метрике $\rho$. 
ДокАЗАТЕЛЬСтво. Положим

$$
\rho_{1}(x, y)=\rho(x, y)+d(f(x), f(y)) .
$$

Ясно, что эта метрика $\rho_{1}$ доставляет тривиальный пример допустимой метрики, относительно которой исходная функция $f$ почти всюду непрерывна. Но по следствию 1 на подходящем множестве $K$ меры $\mu(K)>1-\varepsilon$ из этой непрерывности следует непрерывность функции по $\rho$. Следствие доказано.

Как мы увидим, этот факт не переносится на произвольные функции нескольких аргументов.

\section{2. Определение виртуальной непрерывности и первые примеры.} Для измеримых функций $f(\cdot, \cdot)$ двух переменных аналог теоремы Лузина, т. е. непрерывность на произведении $X^{\prime} \times Y^{\prime}$ множеств меры более $1-\varepsilon$ относительно заданной метрики вида

$$
\rho\left[\left(x_{1}, y_{1}\right),\left(x_{2}, y_{2}\right)\right]=\rho_{X}\left(x_{1}, x_{2}\right)+\rho_{Y}\left(y_{1}, y_{2}\right),
$$

уже выполняется не всегда. Это приводит к следующему понятию, основному в данной работе. (Сумма метрик может быть заменена максимумом или любой другой метрикой, задающей топологию прямого произведения. Чтобы подчеркнуть это обстоятельство, мы обозначаем общую метрику такого класса через $\rho_{X} \times \rho_{Y}$.)

ОПРЕДЕлЕниЕ 2. Измеримая функция $f(\cdot, \cdot)$ на произведении пространств с мерой $(X, \mu) \times(Y, \nu)$ называется собственно виртуально непрерывной, если для любого $\varepsilon>0$ найдутся множества $X^{\prime} \subset X, Y^{\prime} \subset Y$ меры хотя бы $1-\varepsilon$ каждое и допустимые полуметрики $\rho_{X}, \rho_{Y}$ на $X^{\prime}, Y^{\prime}$ соответственно такие, что функция $f$ непрерывна на $\left(X^{\prime} \times Y^{\prime}, \rho_{X} \times \rho_{Y}\right)$. Функция, совпадающая с собственно виртуально непрерывной на множестве полной меры в $X \times Y$, называется виртуально непрерывной. Виртуально непрерывные функции большего числа аргументов определяются точно так же.

В этом определении существенно, что метрика, относительно которой функция должна быть непрерывной, не произвольна, а учитывает структуру прямого произведения (более общо, наличие фиксированных подалгебр - см. далее). Нетрудно убедиться, что не существует универсальной метрики такого типа, т. е. такой метрики, непрерывность в которой равносильна виртуальной непрерывности; это объясняет нетривиальность введенного понятия.

Ясно, что всякая допустимая метрика - как измеримая функция двух переменных - виртуально непрерывна. Функция, непрерывная по какой-либо допустимой метрике указанного вида на произведении пространств, разумеется, виртуально непрерывна. Вырожденные функции (или “функции конечного ранга")

$$
f(x, y)=\sum_{i=1}^{n} \varphi_{i}(x) \psi_{i}(y),
$$

где $\varphi_{i}(\cdot), \psi_{i}(\cdot), i=1, \ldots, n,-$ произвольные измеримые функции, также виртуально непрерывны - достаточно воспользоваться теоремой Лузина для всех функций $\varphi_{i}(\cdot), i=1, \ldots, n$, и $\psi_{i}(\cdot), i=1, \ldots, n$. 
Менее тривиальные примеры виртуально непрерывных функций дают, как мы увидим, функции из некоторых классов Соболева, а также ядра ядерных операторов. Для виртуально непрерывных функций можно корректно определять сужения на некоторые множества нулевой меры - именно, на носители квазибистохастических мер. Об этом см. раздел 3.

Простым примером измеримой функции на квадрате $[0,1]^{2}$, не являющейся виртуально непрерывной, может служить характеристическая функция треугольника $\{x \geqslant y\}$. Вообще, в случае функций двух переменных на группе, которые зависят от "частного" двух аргументов, критерий виртуальной непрерывности прост.

ПредЛОЖениЕ 2. Пусть $G$ - метризуемая компактная группа, $f$ - измеримая по мере Хаара функиия на $G$. Тогда функиия $F(x, y):=f\left(x y^{-1}\right)$ на $G \times G$ виртуально непрерьвна, если и только если функиия $f$ эквивалентна непрерывной.

Подчеркнем, что определение виртуально непрерывных функций не топологическое, а чисто теоретико-мерное, т. е. использует только измеримую структуру пространств и меры на них (иначе говоря, это понятие относится к объектам категории пространств с мерой; более традиционный термин для таких понятий в русской литературе - "метрические понятия", но мы избегаем его из-за нежелательной ассоциации с "метриками": именно от метрики понятие виртуальной непрерывности не зависит). По своей природе это понятие тесно связано с идеей замены метрик на данном пространстве с мерой, о чем шла речь выше. Заметим, что прямой смысл предложения 2 состоит в известном факте о том, что групповая и теоретико-мерная структуры позволяют восстановить топологию.

2.3. Дальнейшие свойства виртуально непрерывных функций. Прежде всего, виртуально непрерывные функции автоматически обладают более сильными свойствами, чем это требуется по определению.

Во-первых, пользуясь следствием 1, немедленно получаем, что метрики можно считать наперед заданными.

Теорема 2. Пусть функиия $f(\cdot, \cdot)$ собственно виртуалъно непреръвна. Тогда для любого $\varepsilon>0$ и любъх допустимых метрик $\rho_{X}, \rho_{Y}$ на $X, Y$ найдутся множества $X^{\prime} \subset X, Y^{\prime} \subset Y$ меры хотя бъ $1-\varepsilon$ каждое такие, что функиия $f$ непрерывна на $\left(X^{\prime} \times Y^{\prime}, \rho_{X} \times \rho_{Y}\right)$.

С другой стороны, выбирая подходящим образом метрики, можно добиться того, чтобы множества $X^{\prime}, Y^{\prime}$ из определения имели полную меру.

Теорема 3. Пусть функиия $f(\cdot, \cdot)$ собственно виртуалъно непреръьна. Тогда найдутся множества $X^{\prime} \subset X, Y^{\prime} \subset Y$ полной меры каждое и допустимъе полуметрики $\rho_{X^{\prime}}, \rho_{Y^{\prime}}$ на $X^{\prime}, Y^{\prime}$ соответственно такие, что функция $f$ непрерьвна на $\left(X^{\prime} \times Y^{\prime}, \rho_{X^{\prime}} \times \rho_{Y^{\prime}}\right)$.

ДокАЗАтЕльство. Зафиксируем допустимые метрики $\sigma_{X}, \sigma_{Y}$ на $X, Y$ соответственно. Для каждого натурального $n$, пользуясь теоремой 2 , найдем множества $X_{n} \subset X$ и $Y_{n} \subset Y$, мера каждого из которых не меньше $1-2^{-n}$, 
такие, что функция $f$ непрерывна на $X_{n} \times Y_{n}$ по метрике $\sigma_{X} \times \sigma_{Y}$. Введем разрезные полуметрики на $X$ :

$$
\rho_{X ; n}\left(x, x^{\prime}\right)= \begin{cases}0, & \text { если } x, x^{\prime} \in X_{n} \text { или } x, x^{\prime} \notin X_{n} ; \\ 1, & \text { если } x \in X_{n}, x^{\prime} \notin X_{n} \text { или } x^{\prime} \in X_{n}, x \notin X_{n} .\end{cases}
$$

Определим множество полной меры $X^{\prime}=\bigcup_{n=1}^{\infty} \bigcap_{k>n} X_{k}$. Метрику $\rho_{X}$ зададим как $\sigma_{X}+\sum_{n} 2^{-n} \rho_{X ; n}$ (несложно видеть, что эта метрика будет допустимой). Аналогично определим множество $Y^{\prime}$ и метрику $\rho_{Y}$.

Докажем, что функция $f(x, y)$ непрерывна на $X^{\prime} \times Y^{\prime}$ по метрике $\rho_{X} \times \rho_{Y}$. Рассмотрим сходящиеся по метрикам $\rho_{X}, \rho_{Y}$ последовательности $x_{n} \rightarrow x_{0}$, $y_{n} \rightarrow y_{0}$ в $X^{\prime}, Y^{\prime}$ соответственно. Пусть $N$ - настолько большое число, что $x_{0} \in X_{N}, y_{0} \in Y_{N}$. Тогда из сходимости по полуметрике $\rho_{X ; n}$ следует, что $x_{n} \in X_{N}$ при всех достаточно больших $n$; аналогично, $y_{n} \in Y_{N}$ при больших $n$. Теперь сходимость $f\left(x_{n}, y_{n}\right) \rightarrow f\left(x_{0}, y_{0}\right)$ следует из непрерывности $f$ на $X_{N} \times Y_{N}$ по метрике $\sigma_{X} \times \sigma_{Y}$. Теорема доказана.

В качестве следствия установим следующее утверждение.

ПрЕДЛОЖЕНИЕ 3. Если функиии $f(x, y), g(x, y)$ собственно виртуалъно непрерывны и совпадают на множестве полной меры в $X \times Y$, то существуют множества полной меры $X^{\prime} \subset X, Y^{\prime} \subset Y$ такие, что $f(x, y)=g(x, y)$ для всех точек $x \in X^{\prime}, y \in Y^{\prime}$.

ДоказАтеЛьСтво. Применим к каждой из функций $f, g$ теорему 3. Соответствующие множества полной меры $X^{\prime} \subset X, Y^{\prime} \subset Y$ можно считать совпадающими (пересечем множества, построенные для $f$ и для $g$ ), равно как и допустимые полуметрики $\rho_{X}, \rho_{Y}$ (сложим полуметрики, построенные для $f$ и для $g$ ). Кроме того, можно считать множества $X^{\prime}, Y^{\prime}$ носителями мер $\left.\mu\right|_{X^{\prime}},\left.\nu\right|_{Y^{\prime}}$. Теперь заметим, что множество точек $(x, y) \in X^{\prime} \times Y^{\prime}$, для которых $f(x, y) \neq$ $g(x, y)$, открыто в $X^{\prime} \times Y^{\prime}$, а значит, либо имеет положительную меру (что невозможно по предположению), либо пусто - что и требовалось. Предложение доказано.

Таким образом, все собственно виртуально непрерывные функции, эквивалентные данной виртуально непрерывной функции, совпадают на произведении множеств полной меры.

Функцию двух переменных на $X \times Y$ полезно рассматривать как отображение из $X$ в пространство функций на $Y$ (т. е. $\left.f(x, y) \equiv f_{x}(y)\right)$. Более подробно о том, как использовать это для классификации измеримых функций, см. в [20]. Виртуальная непрерывность описывается в этих терминах следующим равносильным определением.

Теорема 4. Следующие свойства функиии $f(\cdot, \cdot)$ равносилънъ:

(i) $f$ виртуально непрерывна;

(ii) для любого $\varepsilon>0$ найдутся множества $X^{\prime} \subset X, Y^{\prime} \subset Y$ меры хотя бъ $1-\varepsilon$ каждое и допустимая полуметрика $\rho_{Y}$ на $Y^{\prime}$ такие, что функиия $f_{x}(\cdot)$ эквивалентна непрерывной на $\left(Y^{\prime}, \rho_{Y}\right)$ при почти каждом $x \in X^{\prime}$; 
(iii) для любого $\varepsilon>0$ найдутся множества $X^{\prime} \subset X, Y^{\prime} \subset Y$ меры хотя бы $1-\varepsilon$ каждое такие, что множество функиий вида $f_{x}(\cdot)$ на $Y^{\prime}$ (переменная $x$ пробегает $X^{\prime}$ ) образует вполне ограниченное (предкомпактное) метрическое подпространство в $L_{\infty}\left(Y^{\prime}\right)$;

(iv) для любого $\varepsilon>0$ найдутся множества $X^{\prime} \subset X, Y^{\prime} \subset Y$ меры хотя бъ $1-\varepsilon$ каждое такие, что множество функиий вида $f_{x}(\cdot)$ на $Y^{\prime}$ (переменная $x$ пробегает $X^{\prime}$ ) образует сепарабельное метрическое подпространство в $L_{\infty}\left(Y^{\prime}\right)$.

ДоказАтельство. Ясно, что (i) влечет (ii), а (iii) влечет (iv).

Выведем (iii) из (ii). Удалив из множеств $X^{\prime}$ и $Y^{\prime}$ подходящие множества нулевой меры, мы можем считать, что при каждом $x \in X^{\prime}$ функция $f(x, \cdot)$ эквивалентна непрерывной на $Y^{\prime}$ и при каждом $y \in Y^{\prime}$ функция $f(\cdot, y)$ измерима на $X^{\prime}$. Выберем компактное множество $Y_{1} \subset Y^{\prime}$ такое, что $\nu\left(Y_{1}\right)>1-2 \varepsilon$. Далее, заменим $Y_{1}$ на носитель $\operatorname{supp}\left(\left.\nu\right|_{Y_{1}}\right)$ сужения меры $\nu$ на $Y_{1}$. Теперь для непрерывных функций на $Y_{1}$ расстояния в $C\left(Y_{1}\right)$ и в $L_{\infty}\left(Y_{1}\right)$ совпадают. Пусть $\mathscr{S}$ - счетное семейство открытых шаров в $Y_{1}$, образующих базу топологии. Для $x \in X^{\prime}$ обозначим через $f_{x}^{\prime}(\cdot)$ непрерывную функцию на $Y_{1}$, которой эквивалентна функция $f(x, \cdot)$. Докажем, что отображение $\Phi: x \mapsto f_{x}^{\prime}(\cdot)$ измеримо как отображение из $X$ в $C\left(Y_{1}\right)$ (с борелевской сигма-алгеброй). Для этого достаточно проверить, что прообраз шара

$$
X_{g}:=\left\{x \in X: \forall y \in Y_{1}\left|f_{x}^{\prime}(y)-g(y)\right| \leqslant r\right\}
$$

измерим для любой непрерывной функции $g \in C\left(Y_{1}\right)$ и любого положительного $r$. Заметим, что неравенство $\left|f_{x}^{\prime}(y)-g(y)\right| \leqslant r$ выполняется при всех $y \in Y_{1}$ тогда и только тогда, когда выполняются всевозможные неравенства вида

$$
\frac{1}{\nu(B)}\left|\int_{B} f_{x}^{\prime}(y) d \nu(y)-\int_{B} g(y) d \nu(y)\right| \leqslant r
$$

для всех шаров $B \in \mathscr{S}$. Но под интегралом функцию $f_{x}^{\prime}(\cdot)$ можно заменить на эквивалентную функцию $f(x, \cdot)$, а отображение

$$
x \mapsto \int_{B} f(x, y) d \nu(y)
$$

измеримо по $x$. Итак, множество $X_{g}$ есть пересечение счетного числа измеримых, так что оно измеримо.

Таким образом, отображение $\Phi$ задает борелевскую меру на $C\left(Y_{1}\right)$ (как обратный образ меры $\mu$ на $\left.X^{\prime}\right)$. Пространство $C\left(Y_{1}\right)$ сепарабельно и полно, следовательно, эта мера внутренне регулярна и найдется компакт $K \subset C\left(Y_{1}\right)$ такой, что $\mu\left(\Phi^{-1}(K)\right)>1-2 \varepsilon$. В силу произвольности $\varepsilon$ это доказывает, что функция $f(x, y)$ удовлетворяет (iii).

Осталось доказать виртуальную непрерывность функции $f$ в предположении (iv). Зафиксируем $\varepsilon>0$. Выберем $X^{\prime}, Y^{\prime}$ как в (iv). Сепарабельное семейство функций вида $f(x, \cdot)$ в $L_{\infty}\left(Y^{\prime}\right)$ обозначим $K$. Пусть $K^{\prime}$ - счетное плотное в $K$ семейство функций. Несложно построить допустимую метрику $\rho$ на $Y^{\prime}$, в которой все функции из $K^{\prime}$ непрерывны (можно, например, рассмотреть равномерно сходящийся ряд из метрик, построенных для этих функций по отдельности). Пусть $Y_{1} \subset Y^{\prime}$ - компакт по метрике $\rho$, имеющий меру не 
меньше $1-2 \varepsilon$. При необходимости заменим $Y_{1}$ на носитель сужения меры $\nu$ на $Y_{1}$. Теперь любой открытый шар в $Y_{1}$ имеет положительную меру. Из этого следует, что для непрерывных функций на $Y_{1}$ расстояния в $C\left(Y_{1}\right)$ и в $L_{\infty}\left(Y_{1}\right)$ совпадают. Таким образом, любая функция $f(x, \cdot), x \in X^{\prime}$, эквивалентна на $Y_{1}$ некоторой однозначно определенной непрерывной функции $f^{\prime}(x, \cdot)$. Введем на $X^{\prime}$ полуметрику

$$
\rho_{X^{\prime}}\left(x, x^{\prime}\right)=\left\|f^{\prime}(x, \cdot)-f^{\prime}\left(x^{\prime}, \cdot\right)\right\|_{C\left(Y_{1}\right)} .
$$

Ее допустимость следует из сепарабельности пространства $C\left(Y_{1}\right)$. Функция $f^{\prime}$ будет непрерывна на $X^{\prime} \times Y_{1}$ по $\rho_{X^{\prime}} \times \rho$. В самом деле,

$$
\left|f^{\prime}\left(x_{n}, y_{n}\right)-f^{\prime}\left(x_{0}, y_{0}\right)\right| \leqslant\left|f^{\prime}\left(x_{n}, y_{n}\right)-f^{\prime}\left(x_{0}, y_{n}\right)\right|+\left|f^{\prime}\left(x_{0}, y_{n}\right)-f^{\prime}\left(x_{0}, y_{0}\right)\right|
$$

и при $x_{n} \rightarrow x_{0}, y_{n} \rightarrow y_{0}$ оба слагаемых стремятся к 0 . Функция $f^{\prime}$ собственно виртуально непрерывна на $X^{\prime} \times Y_{1}$. По теореме Фубини она эквивалентна исходной функции $f$ на $X^{\prime} \times Y_{1}$. В силу произвольности $\varepsilon>0$ это доказывает виртуальную непрерывность $f$. Теорема доказана.

Замечательно, что в (ii)-(iv) роль пространств $X$ и $Y$ (т. е. аргументов функции) различна - тем не менее апостериори эти свойства симметричны относительно замены порядка аргументов. В этом также сказывается нетривиальность понятия виртуальной непрерывности.

Из приведенных характеристик виртуальной непрерывности легко вывести, что в пространстве всех измеримых функций двух переменных (с топологией сходимости по мере) совокупность виртуально непрерывных функций образует нигде не плотное множество.

2.4. Виртуальная топология. Как известно, функция непрерывна, если прообраз любого открытого множества открыт. Виртуальную непрерывность функции двух переменных также можно характеризовать подобным образом.

ОПРЕДЕЛЕНИЕ 3. Измеримое множество $Z \subset X \times Y$ называется виртуально открытым, если для некоторых множеств $X^{\prime} \subset X, Y^{\prime} \subset Y$ полной меры множество $Z \cap\left(X^{\prime} \times Y^{\prime}\right)$ есть счетное объединение измеримых прямоугольников $R_{i}=A_{i} \times B_{i}, i=1,2, \ldots$. Множество называется виртуально замкнутым, если его дополнение виртуально открыто.

Смысл (и название) введенного понятия объясняется следующим утверждением.

Лемма 2. 1) Пусть $X^{\prime}, Y^{\prime}$ - множества полной меры в $X, Y$ соответственно, $\rho_{X}, \rho_{Y}$ - допустимые полуметрики на $X^{\prime}, Y^{\prime}$, множество $Z \subset X \times Y$ таково, что $Z \cap X^{\prime} \times Y^{\prime}$ открыто по полуметрике $\rho_{X} \times \rho_{Y}$. Тогда множество $Z$ виртуально открыто.

2) Наоборот, всякое виртуально открытое множество в $X \times Y$ является таковым для подходящих подмножеств $X^{\prime}, Y^{\prime}$ и подходящих допустимых полуметрик $\rho_{X}, \rho_{Y}$.

ДокАЗАТЕЛЬство. 1) Заменяя множества $X^{\prime}, Y^{\prime}$ на подходящие подмножества полной меры, добьемся того, что $\left(X^{\prime}, \rho_{X}\right),\left(Y^{\prime}, \rho_{Y}\right)$ - сепарабельные полуметрические пространства, все шары в которых измеримы по мерам $\mu, \nu$ 
соответственно. Тогда топологии этих пространств имеют счетные базы, состоящие из измеримых множеств, а потому топология прямого произведения имеет счетную базу, состоящую из измеримых прямоугольников. Это и означает, что открытое подмножество в $X^{\prime} \times Y^{\prime}$ есть счетное объединение измеримых прямоугольников, а значит, виртуально открыто.

2) Для любого наперед заданного счетного семейства измеримых подмножеств в $X$ несложно построить допустимую полуметрику, в которой каждое из них будет открытым (достаточно сложить соответствующие разрезные полуметрики с быстро убывающими коэффициентами). Проводя эту процедуру для проекций на $X$ входящих в данное виртуально открытое множество прямоугольников, построим необходимую допустимую полуметрику на $X$; аналогично построим допустимую полуметрику на $Y$.

Лемма доказана.

Теорема 5. Измеримая функиия $f(x, y)$ на $X \times Y$ собственно виртуалъно непрерывна тогда и только тогда, когда $f$-прообраз любого открытого множества на прямой виртуально открыт.

ДокАзАтЕльство. Если функция $f$ собственно виртуальна непрерывна, то по теореме 3 она непрерывна на произведении множеств полной меры $X^{\prime} \times Y^{\prime}$ относительно произведения допустимых полуметрик $\rho_{X} \times \rho_{Y}$. Тогда $f$-прообразы открытых множеств на прямой обладают структурой, описанной в лемме 2.

Пусть теперь $f$-прообраз любого рационального интервала на прямой виртуально открыт. Тогда найдутся множества полной меры $X^{\prime}, Y^{\prime}$ такие, что для сужения $f: X^{\prime} \times Y^{\prime} \rightarrow \mathbb{R}$ все эти прообразы будут счетными объединениями измеримых прямоугольников в $X^{\prime} \times Y^{\prime}$ (в качестве $X^{\prime}, Y^{\prime}$ следует взять пересечения соответствующих множеств полной меры). Рассматривая ряды из разрезных полуметрик, несложно построить допустимые метрики на $X^{\prime}, Y^{\prime}$ такие, что каждый из данного счетного множества прямоугольников будет открытым. Тогда функция $f$ будет непрерывной по паре построенных допустимых полуметрик на $X^{\prime} \times Y^{\prime}$ - т. е. будет собственно виртуально непрерывной. Теорема доказана.

2.5. Толщина. Рассмотрим пространство $X \times Y$ с мерой $\mu \times \nu$; в сигма-алгебре его измеримых множеств естественно выделены две сигма-подалгебры, определяемые проекциями на $X$ и $Y$. Далее мы будет писать $A \stackrel{\bmod 0}{\subset} B$, если $A, B \subset X \times Y$ и $(\mu \times \nu)(B \backslash A)=0$. Кроме того, мы будем писать $\stackrel{\bmod 0}{\leqslant}$ или $\bmod 0$

$\geqslant$, если соответствующее неравенство выполнено $(\mu \times \nu)$-почти всюду.

ОПРЕДЕЛЕНИЕ 4. Для измеримого множества $Z \subset X \times Y$ назовем его собственной толщиной величину

$$
\operatorname{sth}(Z)=\inf \{\mu(\tilde{X})+\nu(\tilde{Y}): \tilde{X} \subset X, \tilde{Y} \subset Y, Z \subset(\tilde{X} \times Y) \cup(X \times \tilde{Y})\} .
$$

Толщиной множества $Z$ назовем величину

$$
\operatorname{th}(Z)=\inf \{\mu(\widetilde{X})+\nu(\widetilde{Y}): \tilde{X} \subset X, \widetilde{Y} \subset Y, Z \stackrel{\bmod 0}{\subset}(\widetilde{X} \times Y) \cup(X \times \tilde{Y})\}
$$

иными словами,

$$
\operatorname{th}(Z)=\min \left\{\operatorname{sth}\left(Z^{\prime}\right): Z^{\bmod 0} Z^{\prime}\right\}
$$


Ясно, что минимум в (2) всегда достигается, поскольку можно рассмотреть пересечение минимизирующей последовательности множеств.

Множества $\widetilde{X} \times Y, X \times \widetilde{Y}$ суть в точности множества из выделенных сигма-подалгебр, что позволяет определить обобщенную толщину для других выделенных сигма-алгебр в стандартном пространстве. Отметим непосредственно вытекающие из определения свойства введенного понятия:

- толщина множества всегда не превосходит 1 и равна 0 в точности для множеств меры 0 ;

- толщина подмножества не превосходит толщины множества;

- толщина множества не меньше его меры;

- толщина конечного или счетного объединения множеств не превосходит суммы толщин.

Несложно устанавливается также следующая лемма.

Лемма 3. Если $Z \subset X \times Y$ - виртуально открытое множество, то $\operatorname{th}(Z)=\operatorname{sth}(Z)$.

ДокАзАТЕЛЬство. Достаточно доказать, что

$$
\operatorname{sth}(Z) \leqslant \mu(\tilde{X})+\nu(\tilde{Y}), \quad \text { если } Z \stackrel{\bmod 0}{\subset}(\tilde{X} \times Y) \cup(X \times \tilde{Y}) .
$$

Переходя к множествам полной меры в $X$ и в $Y$, будем считать, что $Z=$ $\bigcup_{i=1}^{\infty}\left(A_{i} \times B_{i}\right)$. Заметим, что если

$$
A_{i} \times B_{i} \stackrel{\bmod 0}{\subset}(\widetilde{X} \times Y) \cup(X \times \tilde{Y}),
$$

то либо $A_{i} \stackrel{\bmod 0}{\subset} \tilde{X}$, либо $B_{i} \stackrel{\bmod 0}{\subset} \widetilde{Y}$. В обоих случаях можно добавить множества нулевой меры к $\widetilde{X}, \widetilde{Y}$ так, чтобы в (3) можно было заменить $\underset{\subset}{\bmod 0}$ на $\subset$. Сделав так поочередно для всех $i=1,2, \ldots$, получим требуемое. Лемма доказана.

Равносильное и в ряде ситуаций более удобное для применений определение толщины дает следующая лемма.

ЛЕмма 4. Для любого множества $Z$ рассмотрим пары измеримых функицй $f: X \rightarrow[0,1], g: Y \rightarrow[0,1]$ таких, что $f(x)+g(y) \geqslant \chi_{Z}(x, y)$ (coответственно $\left.f(x)+g(y) \stackrel{\bmod 0}{\geqslant} \chi_{Z}(x, y)\right)$. Тогда собственная толщина (соответственно толщина) множества $Z$ есть точная нижняя грань суммы интегралов $\int_{X} f d \mu u \int_{Y} g d \nu$. Кроме того, эта точная ниюняя грань реализуется, как реализуется и инфимум в (1).

ДоказАтельство. Ясно, что если множества $\widetilde{X} \subset X$ и $\widetilde{Y} \subset Y$ таковы, что $Z \subset(\widetilde{X} \times Y) \cup(X \times \widetilde{Y})$, то $f=\chi_{\widetilde{X}}$ и $g=\chi_{\tilde{Y}}$ удовлетворяют условию $f(x)+g(y) \geqslant \chi_{Z}(x, y)$. Таким образом, надо лишь доказать, что если $f$ и $g$ удовлетворяют условию $f(x)+g(y) \geqslant \chi_{Z}(x, y)$, то $\int_{X} f+\int_{Y} g \geqslant \operatorname{sth}(Z)$. Для каждого $t \in[0,1]$ рассмотрим множества

$$
X_{t}=\{x \in X: f(x) \geqslant t\} \quad \text { и } \quad Y_{t}=\{y \in Y: g(y) \geqslant t\} .
$$


Ясно, что если $f(x)+g(y) \geqslant 1$, то для любого $t$ либо $x \in X_{t}$, либо $y \in Y_{1-t}$. Таким образом, для любого $t$ выполнено неравенство $\chi_{Z}(x, y) \leqslant \chi_{X_{t}}(x)+\chi_{Y_{1-t}}(y)$. Стало быть, $\operatorname{sth}(Z) \leqslant \mu\left(X_{t}\right)+\nu\left(Y_{1-t}\right)$. Интегрируя по $t$, получаем:

$$
\operatorname{sth}(Z) \leqslant \int_{0}^{1}\left(\mu\left(X_{t}\right)+\nu\left(Y_{1-t}\right)\right) d t=\int_{X} f+\int_{Y} g .
$$

Кроме того, если $\operatorname{sth}(Z)=\int_{X} f+\int_{Y} g$, то при почти всех $t \in[0,1]$ пара множеств $\left(X_{t}, Y_{1-t}\right)$ доставляет инфимум в $(1)$.

Осталось доказать, таким образом, что существует минимизирующая пара функций. Рассмотрим минимизирующую последовательность пар $\left(f_{n}(x), g_{n}(y)\right)$, $\int f_{n}+\int g_{n} \rightarrow \operatorname{sth}(Z)$. Благодаря известной теореме Комлоша [6] можно считать, что последовательность функций $f_{n}^{\prime}:=\left(f_{1}+\cdots+f_{n}\right) / n$ сходится к некоторой функции $f$ почти всюду в $X$, а $g_{n}^{\prime}:=\left(g_{1}+\cdots+g_{n}\right) / n$ сходится к некоторой функции $g$ почти всюду в $Y$ (здесь используется только простая версия теоремы Комлоша, в которой функции равномерно ограничены). Тогда можно считать, что

$$
\begin{aligned}
& f(x)=\limsup _{n} f_{n}^{\prime}(x) \quad \text { при всех } x \in X, \\
& g(y)=\limsup _{n}^{\prime} g_{n}^{\prime}(y) \quad \text { при всех } y \in Y .
\end{aligned}
$$

Из этого следует, что $f(x)+g(y) \geqslant \chi_{Z}(x, y)$ при всех $x \in X, y \in Y$, так что пара функций $(f, g)$ - минимизирующая. Лемма доказана.

Применяя лемму 4, можно установить "непрерывность толщины снизу".

Лемма 5. Пусть $\left\{Z_{n}\right\}$ - возрастающая последовательность измеримых множеств, $Z=\bigcup_{n} Z_{n}$. Тогда

$$
\operatorname{th}(Z)=\lim \operatorname{th}\left(Z_{n}\right), \quad \operatorname{sth}(Z)=\lim \operatorname{sth}\left(Z_{n}\right) .
$$

ДокАЗАТЕЛЬСтво. Ясно, что для любого $n$ выполнено неравенство $\operatorname{th}(Z) \geqslant$ $\operatorname{th}\left(Z_{n}\right)$, поэтому

$$
\operatorname{th}(Z) \geqslant \lim \operatorname{th}\left(Z_{n}\right) .
$$

Докажем обратное неравенство. Найдем функции $f_{n}: X \rightarrow[0,1], g_{n}: Y \rightarrow[0,1]$ такие, что

$$
f_{n}(x)+g_{n}(y) \stackrel{\bmod 0}{\geqslant} \chi_{Z_{n}}(x, y) \quad \text { и } \int_{X} f_{n}+\int_{Y} g_{n} \leqslant \operatorname{th}\left(Z_{n}\right)+\frac{1}{n} .
$$

Так как из ограниченной последовательности в $L^{2}$ можно выделить слабо сходящуюся подпоследовательность, то, дважды проредив последовательность, мы можем считать, что последовательность функций $f_{n}$ сходится слабо к $f$ в $L^{2}(X, \mu)$, а $g_{n}$ сходится слабо к $g$ в $L^{2}(Y, \nu)$. Тогда $f_{n}(x)+g_{n}(y)$ сходится к $f(x)+g(y)$ слабо в $L^{2}(X \times Y, \mu \times \nu)$. Так как переход к слабому пределу сохраняет неравенства, получаем, что $f: X \rightarrow[0,1]$ и $g: Y \rightarrow[0,1]$. Кроме того, для любого $n$ выполнено неравенство

$$
f(x)+g(y) \stackrel{\bmod 0}{\geqslant} \chi_{Z_{n}}(x, y) .
$$


Стало быть,

$$
f(x)+g(y) \stackrel{\bmod 0}{\geqslant} \chi_{Z}(x, y)
$$

Ho

$$
\int_{X} f+\int_{Y} g=\lim \left(\int_{X} f_{n}+\int_{Y} g_{n}\right) \leqslant \lim \operatorname{th}\left(Z_{n}\right),
$$

поэтому $\operatorname{th}(Z) \leqslant \lim \operatorname{th}\left(Z_{n}\right)$.

Доказательство для собственной толщины отличается тем, что вместо слабой сходимости в $L^{2}$ следует рассматривать сходимость почти всюду, вытекающую из теоремы Комлоша [6]. Лемма доказана.

Отметим, что непрерывность толщины сверху не имеет места: все множества

$$
\left\{(x, y): 0<|x-y|<\frac{1}{n}\right\} \subset[0,1]^{2}
$$

имеют толщину 1 , но их пересечение пусто.

Определим сходимость функций "по толщине” по аналогии со сходимостью по мере. Это сходимость в метризуемой топологии, задаваемой таким расстоянием.

ОПРЕДЕЛЕниЕ 5. Расстояние $\tau(f(\cdot, \cdot), g(\cdot, \cdot))$ между двумя произвольными измеримыми функциями двух переменных зададим как точную нижнюю грань тех $\varepsilon>0$, для которых

$$
\operatorname{th}(\{(x, y):|f(x, y)-g(x, y)|>\varepsilon\}) \leqslant \varepsilon .
$$

Сходимость по введенной метрике $\tau$ влечет сходимость по мере (но не наоборот).

Лемма 6. Множество измеримых функций полно по метрике $\tau$.

ДокАЗАтЕльство. Предположим, что последовательность $\left\{f_{n}(\cdot, \cdot)\right\}$ измеримых функций фундаментальна по метрике $\tau$. Переходя к подпоследовательности, будем считать, что $\left\|f_{n}-f_{n+1}\right\|_{\tau}<2^{-n}$. Положим

$$
Z_{n}=\left\{(x, y):\left|f_{n}(x, y)-f_{n+1}(x, y)\right|>2^{-n}\right\} .
$$

Тогда $\operatorname{th}\left(Z_{n}\right) \leqslant 2^{-n}$ и для множества $Z_{n}^{\prime}:=\bigcup_{k \geqslant n} Z_{k}$ имеем $\operatorname{th}\left(Z_{n}^{\prime}\right) \leqslant 2^{1-n}$. Следовательно, множество $\bigcap Z_{n}^{\prime}$ имеет нулевую толщину, а вне его последовательность $f_{n}$ сходится поточечно к некоторой функции $f_{0}$. При этом вне множества $Z_{n}^{\prime}$ последовательность функций $f_{n}$ сходится равномерно и

$$
\left|f_{0}-f_{n}\right| \leqslant\left|f_{n}-f_{n+1}\right|+\left|f_{n+1}-f_{n+2}\right|+\cdots \leqslant 2^{1-n} .
$$

Это означает, что $\left\|f_{0}-f_{n}\right\|_{\tau} \leqslant 2^{1-n}$, откуда следует, что $f_{n}$ сходится к $f_{0}$ по метрике $\tau$. Лемма доказана.

Пусть

$$
\xi_{X}: X=\bigsqcup_{i=1}^{n} X_{i}, \quad \xi_{Y}: Y=\bigsqcup_{i=1}^{m} Y_{i}
$$

- конечные разбиения пространств $X, Y$ соответственно на измеримые подмножества положительной меры. Функции, постоянные $\bmod 0$ на каждом из 
прямых произведений $X_{i} \times Y_{j}$, будем называть ступенчатыми функииями. Конечные линейные комбинации

$$
\sum_{i=1}^{N} a_{i}(x) b_{i}(y)
$$

будем называть функщиями конечного ранга.

Связь с виртуальной непрерывностью описывает следующая теорема.

ТЕОРема 6. Замыкания по метрике $\tau$ множества ступенчатых функиий (или множества функций конечного ранга) есть в точности множество виртуально непрерьвных функций. Иными словами, функиия $f$ виртуально непрерывна на $X \times Y$ тогда и только тогда, когда при всяком $\varepsilon>0$ найдутся такие наборы непересекающихся измеримых множеств $A_{1}, \ldots, A_{n} \subset X$, $B_{1}, \ldots, B_{n} \subset Y$ и числа $c_{i j}, 1 \leqslant i, j \leqslant n$, что

$$
\begin{gathered}
\sum \mu\left(A_{i}\right)>1-\varepsilon, \quad \sum \nu\left(B_{i}\right)>1-\varepsilon, \\
\left|F(x, y)-c_{i j}\right|<\varepsilon \quad \text { для почти всех } x \in A_{i}, y \in B_{j} .
\end{gathered}
$$

ДокАзАтельство. Сначала докажем, что если функция $f$ является пределом в метрике $\tau$ последовательности ступенчатых функций $f_{n}$, то она виртуально непрерывна. Выберем допустимые метрики $\rho_{X}$ и $\rho_{Y}$ на $X$ и $Y$ соответственно таким образом, чтобы ступенчатые функции $f_{n}$ были непрерывными.

Проредив последовательность $f_{n}$, мы можем считать, что $\tau\left(f_{n}, f\right)<1 / 2^{n}$. Это значит, что

$$
\operatorname{th}\left(\left\{(x, y):\left|f(x, y)-f_{n}(x, y)\right|>\frac{1}{2^{n}}\right\}\right)<\frac{1}{2^{n}} .
$$

Выберем множества $X_{n} \subset X, Y_{n} \subset Y$ так, что

$$
\left\{(x, y):\left|f(x, y)-f_{n}(x, y)\right|>\frac{1}{2^{n}}\right\} \stackrel{\bmod 0}{\subset}\left(X \times Y_{n}\right) \cup\left(X_{n} \times Y\right)
$$

и $\mu\left(X_{n}\right)+\nu\left(Y_{n}\right)<1 / 2^{n}$. Положим

$$
\tilde{X}_{n}=X \backslash \bigcup_{k>n} X_{k} \quad \text { и } \quad \tilde{Y}_{n}=Y \backslash \bigcup_{k>n} Y_{k} .
$$

Ясно, что $\mu\left(\widetilde{X}_{n}\right)>1-1 / 2^{n}$ и $\nu\left(\widetilde{Y}_{n}\right)>1-1 / 2^{n}$. Сузив, если потребуется, множество $\widetilde{X}_{n}$, мы получим, что оно компактно в метрике $\rho_{X}$, совпадает с носителем сужения меры $\mu$ на $\widetilde{X}_{n}$ и $\mu\left(\widetilde{X}_{n}\right)>1-1 / 2^{n}$. Аналогичную процедуру проведем с множеством $\widetilde{Y}_{n}$. Теперь на $\widetilde{X}_{n} \times \widetilde{Y}_{n}$ расстояния между непрерывными функциями в пространствах $L_{\infty}$ и $C$ совпадают, поэтому $f_{k}-$ сходящаяся в $C\left(\widetilde{X}_{n} \times \widetilde{Y}_{n}\right)$ последовательность функций. Стало быть, функция $f$ эквивалентна непрерывной на $\widetilde{X}_{n} \times \widetilde{Y}_{n}$. В силу произвольности $n$ это означает, что функция $f$ виртуально непрерывна.

Докажем обратное. Пусть функция $f$ виртуально непрерывна. Нам нужно доказать, что она приближается по метрике $\tau$ ступенчатыми функциями. Выберем любое $\varepsilon>0$ и найдем множества $\widetilde{X} \subset X, \widetilde{Y} \subset Y$ и допустимые метрики $\rho_{X}, \rho_{Y}$ такие, что $f$ эквивалентна функции $\widetilde{f}$, непрерывной на $\widetilde{X} \times \widetilde{Y}$, и $\mu(\widetilde{X})>1-\varepsilon, \nu(\widetilde{Y})>1-\varepsilon$. Сузив, если это потребуется, множества $\widetilde{X}$ и $\widetilde{Y}$, их 
можно считать компактами в соответствующих метриках. Осталось воспользоваться равномерной непрерывностью функции $\widetilde{f}$ на компакте и разбить $\widetilde{X}$ и $\widetilde{Y}$ на достаточно маленькие части так, чтобы функция $\widetilde{f}$ была с точностью до $\varepsilon$ постоянной на произведениях элементов разбиений. Таким образом, мы найдем ступенчатую функцию, расстояние от которой до $f$ в метрике $\tau$ не превосходит $\varepsilon$. Теорема доказана.

Это рассуждение выявляет чисто метрический характер понятия виртуальной непрерывности, а также показывает возможность определения этого понятия для произвольной пары сигма-подалгебр. Относительно близких понятий см. также [12].

В качестве приложения теоремы 6 докажем предложение 2.

ДОКАЗАТЕЛЬСТво ПРЕДЛОЖЕНИЯ 2. Если функция $f$ эквивалентна непрерывной, то эквивалентной непрерывной по (допустимой относительно меры Хаapa) метрике группы $G$ будет и функция $F$ на $G \times G$; тем более, она виртуально непрерывна.

Докажем обратное. Зафиксируем $\varepsilon>0$. Выберем произвольную точку $g_{0} \in G$ и докажем, что в достаточно малой ее окрестности существенная вариация функции $f$ не превосходит $\varepsilon$. В силу произвольности точки $g_{0}$ и $\varepsilon>0$ получим, что функция $f$ почти всюду совпадает со своим существенным верхним пределом, каковой есть непрерывная функция.

Воспользовавшись виртуальной непрерывностью функции $f$, найдем такие наборы непересекающихся измеримых множеств $A_{1}, \ldots, A_{n}, B_{1}, \ldots, B_{n}$ и числа $c_{i j}, 1 \leqslant i, j \leqslant n$, что

$$
\begin{gathered}
\sum\left|A_{i}\right|>\frac{1}{2}, \quad \sum\left|B_{i}\right|>\frac{1}{2}, \\
\left|F(x, y)-c_{i j}\right|<\frac{\varepsilon}{3} \quad \text { для почти всех } x \in A_{i}, y \in B_{j} .
\end{gathered}
$$

По принципу Дирихле найдутся такие индексы $i, j$, что $|C|>0$, где $C=$ $A_{i} \cap g_{0} B_{j}$. Из непрерывности сдвига в среднем следует, что для некоторой окрестности единицы $\Delta$ выполнено неравенство $|C \cap s C|>|C| / 2$ при всех $s \in \Delta$. Достаточно доказать, что в окрестности $\Delta g_{0}$ точки $g_{0}$ для почти всех точек $z$ выполнено неравенство $\left|f(z)-c_{i j}\right|<\varepsilon / 3$. Пусть $S g_{0}$, где $S \subset \Delta,-$ множество тех $z$, для которых это не так. Тогда для почти всех $x \in C \subset A_{i}, y \in g_{0}^{-1} C \subset B_{j}$ имеем $x y^{-1} \notin S g_{0}$. Иными словами, следующий интеграл равен 0 :

$$
\begin{aligned}
0 & =\int \chi_{C}(x) \chi_{g_{0}^{-1} C}(y) \chi_{S g_{0}}\left(x y^{-1}\right) d x d y \\
& =\int \chi_{C}(x) \chi_{t C}(x) \chi_{S}(t) d x d t=\int_{S}|C \cap t C| d t \geqslant|S| \cdot \frac{|C|}{2},
\end{aligned}
$$

откуда $|S|=0$, что и требовалось (второе равенство соответствует замене переменных $\left.(x, y) \mapsto(x, t), t=x y^{-1} g_{0}^{-1}\right)$. Предложение 2 доказано.

Измеримые функции $f(\cdot, \cdot)$, как мы видели, классифицируются матричными распределениями, т. е. мерами на пространстве бесконечных матриц $\left(a_{i j}\right)_{i, j=1}^{\infty}$, индуцируемых отображением $f \mapsto\left(a_{i j}=f\left(x_{i}, y_{j}\right)\right)$; точки $x_{i}$ в $X$ и $y_{i}$ в $Y$, $i=1,2, \ldots$, выбираются независимо. В этих терминах можно охарактеризовать свойство виртуальной непрерывности. 
Теорема 7. Пусть точки $x_{1}, x_{2}, \ldots$ выбираются случайно и независимо в $X$, а точки $y_{1}, y_{2}, \ldots$ - случайно и независимо в $Y$. Виртуальная непреръвность функиии $f(x, y)$ равносильна каждому из двух следующих свойств.

(i) Для любого $\varepsilon>0$ найдется натуральное $N$ такое, что вероятность следующего события стремится $\kappa 1$ при $n \rightarrow+\infty$ :

если точки $x_{1}, \ldots, x_{n}$ выбираются случайно и независимо в $X$, а точки $y_{1}, \ldots, y_{n}$ - случайно и независимо в $Y$, то найдутся такие разбиения множества индексов,

$$
\{1, \ldots, n\}=\bigsqcup_{i=0}^{N} A_{i}=\bigsqcup_{i=0}^{N} B_{i},
$$

чmo

$$
\left|A_{0}\right|<\varepsilon n, \quad\left|B_{0}\right|<\varepsilon n, \quad\left|f\left(x_{s}, y_{t}\right)-f\left(x_{r}, y_{p}\right)\right|<\varepsilon
$$

nрu $s, r \in A_{i}, p, t \in B_{j}, 0<i, j \leqslant N$.

(ii) Для любого $\varepsilon>0$ найдется натуральное $N$ такое, что вероятность следующего события равна 1:

если точки $x_{1}, x_{2}, \ldots$ выбираются случайно и независимо в $X$, а точки $y_{1}, y_{2}, \ldots$ - случайно и независимо в $Y$, то найдутся такие разбиения натурального ряда,

$$
\{1,2, \ldots\}=\bigsqcup_{i=0}^{N} A_{i}=\bigsqcup_{i=0}^{N} B_{i},
$$

что верхняя плотность множества $A_{0} \cup B_{0}$ (m.e. $\lim \sup \mid\left(A_{0} \cup B_{0}\right) \cap$ $[1, n] \mid / n)$ меньше $\varepsilon$ и

$$
\left|f\left(x_{s}, y_{t}\right)-f\left(x_{r}, y_{p}\right)\right|<\varepsilon \quad \text { npu } s, r \in A_{i}, p, t \in B_{j}, 0<i, j \leqslant N .
$$

ДокАзАтЕльство. Виртуально непрерывная функция аппроксимируется ступенчатыми в метрике $\tau$, а потому удовлетворяет свойствам (i), (ii) в силу закона больших чисел.

Докажем (i), предполагая (ii). Множество, верхняя плотность которого меньше $\varepsilon$, будет содержать менее $2 \varepsilon n$ элементов от 1 до $n$ при всех достаточно больших $n$. Это значит, что с вероятностью 1 при всех достаточно больших $n$ будут существовать разбиения множества $\{1, \ldots, n\}$, удовлетворяющие (i). Но тогда, конечно, и вероятность одного такого события как функция от $n$ стремится к 1.

Осталось вывести виртуальную непрерывность из (i). Будем считать $X, Y$ единичными отрезками $[0,1]$.

Нам понадобится следующая стандартная лемма об оценке больших уклонений $U$-статистик.

Лемма 7. Пусть $Z \subset X \times Y$ - измеримое подмножество в $X \times Y$. Тогда вероятность того, что количество точек вида $\left(x_{i}, y_{j}\right), 1 \leqslant i, j \leqslant n$, в множестве $Z$ отличается от $n^{2}|Z|$ больше, чем на $n^{9 / 5}$, не превосходит $2 n^{-3 / 5}$.

ДоказАтельство. Введем функцию $g(x, y)=\chi_{Z}(x, y)-|Z|$ (с нулевым средним) и случайные величины $\xi_{i, j}=g\left(x_{i}, y_{j}\right)$. У нас будет $n^{2}$ центрированных 
случайных величин, принимающих значения в отрезке $[-1,1]$. Большинство пар этих случайных величин независимы, что позволяет оценить дисперсию:

$$
\begin{aligned}
\mathrm{E}\left(\sum_{i, j} g\left(x_{i}, y_{j}\right)\right)^{2}=\mathrm{E}\left(\sum_{i, j} g\left(x_{i}, y_{j}\right)^{2}+\sum_{\substack{i, j, k \\
k \neq j}} g\left(x_{i}, y_{j}\right) g\left(x_{i}, y_{k}\right)\right. \\
\left.+\sum_{\substack{i, j, k \\
k \neq i}} g\left(x_{i}, y_{j}\right) g\left(x_{k}, y_{j}\right)\right) \leqslant 2 n^{3} .
\end{aligned}
$$

Требуемая оценка теперь следует из неравенства Чебышёва. Лемма доказана.

Выберем подмножество $\mathscr{N} \subset \mathbb{N}$ так, что

$$
\sum_{n \in \mathscr{N}} n^{-3 / 5}<+\infty .
$$

Тогда по лемме Бореля-Кантелли для любого измеримого множества $Z \subset[0,1]^{2}$ и для почти всех случайных пар последовательностей $\left(\left\{x_{i}\right\},\left\{y_{i}\right\}\right)$ при достаточно больших $n \in \mathscr{N}$ выполнены неравенства

$$
\frac{n^{2}}{2}|Z| \leqslant \#\left\{(i, j): 1 \leqslant i, j \leqslant n, \quad\left(x_{i}, y_{j}\right) \in Z\right\} \leqslant 2 n^{2}|Z| .
$$

Применив это рассуждение для счетного набора множеств вида $Z=R \backslash$ $f^{-1}(\Delta)$, где $R$ пробегает все прямоугольники с рациональными координатами вершин, а $\Delta$ пробегает все интервалы вещественной прямой с рациональными концами, получим, что для почти каждой случайной пары последовательностей $\left(\left\{x_{i}\right\},\left\{y_{i}\right\}\right)$ и для каждого $Z$ указанного вида при достаточно больших (зависит от случайной пары и от множества $Z) n \in \mathscr{N}$ выполнено неравенство (4). В дальнейшем мы будем рассматривать только такие пары последовательностей, для которых это свойство выполнено (таковы почти все случайные пары последовательностей).

Зафиксируем $\varepsilon>0$ и найдем по нему $N$ из условия (i). Рассмотрим случайную пару последовательностей независимых точек $x_{1}, x_{2}, \ldots \in X$ и $y_{1}, y_{2}, \ldots \in Y$. $\mathrm{C}$ вероятностью 1 для этой последовательности при каждом $n$ выполнено условие (i). Для каждого $n \in \mathscr{N}$ рассмотрим эмпирические распределения

$$
\mu_{j}(n)=n^{-1} \sum_{i \in A_{j}^{n}} \delta\left(x_{i}\right)
$$

на $X$ и аналогичные эмпирические распределения на $Y$. Переходя, если потребуется, к подпоследовательности, мы можем получить, что последовательности мер $\left\{\mu_{j}(n)\right\}_{n \in \mathscr{N}}$ слабо сходятся при $n \rightarrow+\infty$ к некоторым мерам $\mu_{j}(\infty)$. Можно также считать, что соответствующие меры для $Y$ тоже сходятся. Отметим, что

$$
\sum_{j=1}^{N} \mu_{j}(\infty)=\mu
$$

с вероятностью 1 , так что с вероятностью 1 все меры $\mu_{j}(\infty)$ абсолютно непрерывны по $\mu$, тогда по теореме Радона-Никодима они имеют неотрицательные измеримые плотности $\varphi_{j}$. Имеем $\int \varphi_{0} \leqslant \varepsilon$, так что мера тех $x \in X$, 
для которых $\varphi_{0}(x) \geqslant 1 / 2$, не превосходит $2 \varepsilon$. Для всех остальных $x$ имеем $\sum_{j=1}^{N} \varphi_{j}(x) \geqslant 1 / 2$, поэтому $\varphi_{j}(x) \geqslant 1 /(2 N)$ для некоторого индекса $j$. Таким образом, можно разбить $X$ на множества $X_{0}, X_{1}, \ldots, X_{N}$ такие, что $\mu\left(X_{0}\right)<2 \varepsilon$ и $\varphi_{j} \geqslant 1 /(2 N)$ на $X_{j}$. Аналогичное разбиение построим на $Y$. Докажем, что с вероятностью 1 функция $f$ имеет существенную вариацию (определяемую как существенный супремум минус существенный инфимум) не более $2 \varepsilon$ на $X_{j} \times Y_{k}$ при $j, k \geqslant 1$. Действительно, если существенная вариация больше $2 \varepsilon$, то найдутся рациональные интервалы $\Delta_{1}, \Delta_{2}$, расстояние между которыми не меньше $3 \varepsilon / 2$, такие, что множества $f^{-1}\left(\Delta_{i}\right), i=1,2$, имеют пересечение положительной меры с $X_{j} \times Y_{k}$. Зафиксируем маленькое число $\delta>0$ (будет выбрано позже, зависит от $\varepsilon$ и $N$ ). Тогда можно выделить прямоугольники $R_{i}, i=1,2$, с рациональными координатами вершин такие, что

$$
\left|R_{i} \cap\left(X_{j} \times Y_{k}\right) \cap f^{-1}\left(\Delta_{i}\right)\right|>(1-\delta)\left|R_{i}\right| .
$$

Из слабой сходимости мер, неравенств вида $\varphi_{j} \geqslant 1 /(2 N)$ на $X_{j}$ и аналогичных неравенств на $Y_{k}$ следует, что при $n \rightarrow \infty, n \in \mathscr{N}$,

$$
\begin{aligned}
\left(\mu_{j}(n) \times \nu_{j}(n)\right)\left(R_{i}\right) & \rightarrow\left(\mu_{j}(\infty) \times \nu_{j}(\infty)\right)\left(R_{i}\right) \\
& \geqslant\left(\mu_{j}(\infty) \times \nu_{j}(\infty)\right)\left(R_{i} \cap\left(X_{j} \times Y_{k}\right)\right) \geqslant \frac{1}{4 N^{2}}\left|R_{i} \cap\left(X_{j} \times Y_{k}\right)\right|,
\end{aligned}
$$

поэтому, при достаточно больших $n \in \mathscr{N}$, количество точек вида $\left(x_{s}, y_{t}\right) \in R_{i}$ таких, что $s \in A_{j}^{n}, t \in B_{k}^{n}$, не меньше, чем

$$
\frac{1-\delta}{10 N^{2}}\left|R_{i}\right| n^{2}
$$

В силу свойства (i) либо все эти точки (для $i=1$ и $i=2$ вместе) не лежат в $f^{-1}\left(\Delta_{1}\right)$, либо все они не лежат в $f^{-1}\left(\Delta_{2}\right)$. Но для каждого из множеств $Z=R_{i} \backslash f^{-1}\left(\Delta_{i}\right)$, мера которого не превосходит $\delta\left|R_{i}\right|$, при больших $n \in \mathscr{N}$ количество точек вида $\left(x_{s}, y_{t}\right), 1 \leqslant s, t \leqslant n$, попадающих в него, не превосходит $2 \delta\left|R_{i}\right| n^{2}$, что противоречит полученной оценке при достаточно малых $\delta$.

Таким образом, с вероятностью 1 функция $f$ приближается в метрике $\tau$ ступенчатой, подчиненной построенным разбиениям. Осталось воспользоваться произвольностью $\varepsilon$ и теоремой 6 . Теорема 7 доказана.

2.6. Бистохастические меры и полиморфизмы. С точки зрения теории меры сама по себе функция $k$ аргументов на прямом произведении стандартных пространств с непрерывной мерой есть снова функция на стандартном пространстве с непрерывной мерой - в силу изоморфизма всех таких пространств. Чтобы рассматривать ее именно как функцию $k$ переменных, следует ввести несколько иную категорию, чем категория пространств с мерой.

Именно, рассмотрим пространство с мерой $(\mathscr{X}, \mathfrak{A}, m)$, в сигма-алгебре которого выделено $k$ сигма-подалгебр $\mathfrak{A}_{1}, \ldots, \mathfrak{A}_{k}$. Естественно считать, что эти подалгебры в совокупности порождают всю сигма-алгебру и других требований на эти подалгебры нет. В таком пространстве сомножитель $\mathfrak{A}_{i}$ отождествляется с подалгеброй алгебры $\mathfrak{A}=\prod \mathfrak{A}_{i}$, получающейся умножением этого сомножителя на тривиальные подалгебры остальных сомножителей. Иными словами, 
функции $f\left(x_{1}, \ldots, x_{k}\right)$ на $\mathscr{X}$, измеримые относительно $i$-й сигма-подалгебры $\mathfrak{A}_{i}$, суть функции, зависящие только от одного аргумента $x_{i}(i=1, \ldots, k)$. Точно так же можно определить функции, зависящие от любого меньшего числа переменных. Тем самым, измеримая функция нескольких переменных и сами переменные определяются в пространстве с мерой, в котором выделено семейство сигма-подалгебр, порождающих в совокупности всю сигма-алгебру.

ОПРЕДЕЛЕНИЕ 6. Измеримую функцию на пространстве $\mathscr{X}$ с $k$ выделенными подалгебрами будем называть общей измеримой функиией $k$ аргументов.

В классическом случае эти подалгебры независимы (и сами переменные также называются независимыми, хотя это лишь удачное совпадение двух различных пониманий одного и того же термина "независимость" - вероятностного и аналитического), однако многие факты об измеримых функциях нескольких переменных остаются верными и в общем случае.

Рассмотрим меру $\lambda$, заданную на сигма-алгебре $\mathfrak{A}$. Ее можно ограничивать на сигма-подалгебры $\mathfrak{A}_{i}, i=1, \ldots, k$. Будем рассматривать такие меры $\lambda$, что эти ограничения абсолютно непрерывны относительно меры $m$, суженной на $\mathfrak{A}_{i}$. Если ограничения меры $\lambda$ на сигма-алгебры $\mathfrak{A}_{i}, i=1, \ldots, k$, совпадают с $m$, то такая мера называется мультистохастической мерой относительно данных подалгебр (при $k=2$ - бистохастической); если же эти ограничения лишь эквивалентны $m$ для $i=1, \ldots, k$, то мы называем меру $\lambda$ почти мультистохастической. Наконец, если $\lambda(U) \leqslant m(U)$ для любого $U \in \mathfrak{A}_{i}, i=1, \ldots, k$, меру будем называть субмультистохастической.

Разумеется, бистохастическая мера может быть сингулярной относительно произведения мер. Например, в случае прямого произведения отрезков $(X, \mu)=(Y, \nu)=[0,1]$ бистохастической будет мера $\lambda$, сосредоточенная на диагонали $\{x=y\}$ (образ меры $\mu$ при отображении $x \mapsto(x, x))$.

Во всем дальнейшем мы для простоты полагаем, что $k=2$, т. е. рассматриваем случай двух аргументов, однако никаких серьезных изменений для случая произвольного $k>2$ в наших рассмотрениях и результатах делать не придется. Мы будем рассматривать не только случай независимых аргументов, т. е. не обязательно функции, заданные на прямом произведении двух пространств с непрерывной мерой, - большая часть понятий может быть определена для произвольной пары сигма-подалгебр. Но и случай независимых аргументов часто полезнее рассматривать как общий.

Бистохастические меры на прямом произведении пространств определяют так называемый полиморфизм пространства $(X, \mu)$ в пространство $(Y, \nu)$ (см. [17]), т. е. "многозначное отображение" с инвариантной мерой. Особенно важен случай отождествленных аргументов: $(X, \mathfrak{A}, \mu)=(Y, \mathfrak{B}, \nu)$; тогда полиморфизм обобщает понятие автоморфизма пространства с мерой. Почти бистохастическая мера определяет полиморфизм с квазиинвариантной мерой. Бистохастическая или почти бистохастическая мера $\lambda$ определяет также билинейную (в общем случае $k$-линейную) форму

$$
(f(x), g(y)) \mapsto \int f(x) g(y) d \lambda(x, y),
$$

которой отвечает так называемый марковский (соответственно квазимарковский) оператор в соответствующих функциональных пространствах. Заметим 
лишь, что этот оператор $U_{\lambda}$ есть сжатие (т. е. его норма не превышает единицы), он сохраняет инвариантным конус неотрицательных функций. В случае бистохастической меры этот оператор, как и его сопряженный, оставляет неподвижными константы: $U_{\lambda} 1=1$. О многочисленных связях теории полиморфизмов (марковские операторы, джойнинги, бирасслоения, соответствия, меры Юнга, каплинги и т. д.) см. в [15], [17], [18]. Бистохастические меры играют ключевую роль в бурно развивающейся теории непрерывных графов [7].

Заметим, что для квазибистохастической меры $\lambda$ на $X \times Y$ все множества нулевой собственной толщины измеримы (и имеют меру 0). Тогда и виртуально открытые множества также $\lambda$-измеримы, а потому измеримы собственно виртуально непрерывные функции. Эквивалентные же (по мере $\mu \times \nu$ ) собственно виртуально непрерывные функции эквивалентны и по мере $\lambda$ в силу предложения 3. Таким образом, классу эквивалентности измеримых виртуально непрерывных функиий однозначно соответствует класс $\lambda$-эквивалентности $\lambda$-измеримых функиий. В дальнейшем мы оформим этот тезис как теорему вложения нормированных пространств.

2.7. Норма в пространстве виртуально непрерывных функций. Мы определили сходимость по метрике $\tau$, являющуюся для виртуально непрерывных функций аналогом сходимости по мере. Можно ввести аналоги известных банаховых пространств измеримых функций.

Измеримую функцию $h(\cdot, \cdot)$ на пространстве $(X \times Y, \mu \times \nu)$ назовем суббистохастической, если мера с плотностью $|h(\cdot, \cdot)|$ относительно продакт-меры на $X \times Y$ является суббистохастической. Множество суббистохастических функций обозначим через $\mathscr{S}$.

Назовем функции вида $f(x, y)=a(x)+b(y)$ сепаратными. Следующая конструкция определяет норму (так называемую норму с регулятором) функций двух переменных, где регулятор - сепаратная функция, а норма берется в $L^{1}$. Определим конечную или бесконечную норму измеримой функции $f(\cdot, \cdot)$ равенством

$$
\begin{aligned}
\|f\|_{\mathrm{SR}^{1}}:=\inf \left\{\int_{X} a(x) d \mu(x)+\int_{Y} b(y) d \nu(y):\right. \\
\quad a(x) \geqslant 0, b(y) \geqslant 0,|f(x, y)| \stackrel{\bmod 0}{\leqslant} a(x)+b(y)\} .
\end{aligned}
$$

Связь введенной $\mathrm{SR}^{1}$-нормы и метрики $\tau$ дает следующая лемма.

ЛЕмма 8. Для любой функиии $f$ выполнено неравенство

$$
\tau(0, f) \leqslant \sqrt{2\|f\|_{\mathrm{SR}^{1}}} .
$$

ДокАЗАТЕЛьство. Если $\|f\|_{\mathrm{SR}^{1}}=\infty$, то утверждение тривиально. Пусть $\|f\|_{\mathrm{SR}^{1}}<t^{2} / 2$ для некоторого $t>0$. Тогда найдутся неотрицательные функции $a: X \rightarrow \mathbb{R}$ и $b: Y \rightarrow \mathbb{R}$ такие, что

$$
|f(x, y)| \stackrel{\bmod 0}{\leqslant} a(x)+b(y) \quad \text { и } \quad \int_{X} a+\int_{Y} b<\frac{t^{2}}{2} .
$$


Но тогда по неравенству Чебышёва имеем

$$
\mu\left\{x: a(x) \geqslant \frac{t}{2}\right\}+\nu\left\{y: b(y) \geqslant \frac{t}{2}\right\}<t .
$$

Ho

$$
\{(x, y): f(x, y) \geqslant t\} \stackrel{\bmod 0}{\subset}\left\{x: a(x) \geqslant \frac{t}{2}\right\} \times Y \cup X \times\left\{y: b(y) \geqslant \frac{t}{2}\right\},
$$

поэтому $\tau(0, f)<t$, что и доказывает требуемое неравенство. Лемма доказана.

СледСтвиЕ 3. Сходимость в $\mathrm{SR}^{1}$-норме влечет сходимость в метрике $\tau$.

Следующая теорема есть аналог известной теоремы двойственности Л. В. Канторовича [4] в транспортной задаче - именно, двойственности между пространством мер с метрикой Канторовича и пространством функций Липшица (см. также [21]).

ТеОрема 8. Имеет место формула

$$
\|f\|_{\mathrm{SR}^{1}}=\sup \left\{\int_{X \times Y}|f(x, y)| h(x, y) d \mu(x) d \nu(y): h \in \mathscr{S}\right\} .
$$

ДокАЗАтЕльство. Покажем сначала, что левая часть (5) не меньше правой. Действительно, если $h \in \mathscr{S}$, а функции $a: X \rightarrow \mathbb{R}$ и $b: Y \rightarrow \mathbb{R}$ таковы, что $|f(x, y)| \stackrel{\bmod 0}{\leqslant} a(x)+b(y)$, то

$$
\begin{aligned}
\int_{X \times Y} & |f(x, y) h(x, y)| d \mu(x) d \nu(y) \leqslant \int_{X \times Y}|h(x, y)|(a(x)+b(y)) d \mu(x) d \nu(y) \\
& \leqslant \int_{X} a(x) \int_{Y}|h(x, y)| d \nu(y) d \mu(x)+\int_{Y} b(y) \int_{X}|h(x, y)| d \mu(x) d \nu(y) \\
& \leqslant \int_{X} a(x) d \mu(x)+\int_{Y} b(y) d \nu(y) .
\end{aligned}
$$

Переходя к инфимуму по допустимым парам функций $a$ и $b$, получаем искомое неравенство.

Осталось доказать, что правая часть (5) не меньше левой. Выберем произвольное $\theta>0$ и рассмотрим два выпуклых подмножества в $L^{1}(X \times Y, \mu \times \nu)$ :

$$
\begin{aligned}
& A=\left\{a(x)+b(y): a, b \geqslant 0, \int_{X} a(x) d \mu(x)+\int_{Y} b(y) d \nu(y) \leqslant\|f\|_{\mathrm{SR}^{1}}-\theta\right\}, \\
& B=\{g(x, y): g(x, y) \stackrel{\bmod 0}{\geqslant}|f(x, y)|\} .
\end{aligned}
$$

По определению нормы эти два множества не пересекаются. Докажем, что расстояние между этими множествами в $L^{1}$ положительно. Если это не так, то найдутся последовательности неотрицательных функций $a_{n}, b_{n}, g_{n}$ такие, что $a_{n}(x)+b_{n}(y) \in A, g_{n} \in B$ и

$$
\left\|a_{n}(x)+b_{n}(y)-g_{n}(x, y)\right\|_{L^{1}(X \times Y)} \rightarrow 0 .
$$

Переходя, если нужно, к подпоследовательности, мы можем получить, что

$$
a_{n}(x)+b_{n}(y)-g_{n}(x, y) \rightarrow 0 \quad(\mu \times \nu) \text {-почти всюду. }
$$


Воспользуемся теоремой Комлоша [6]. Переходя к соответствующей подпоследовательности, можно добиться того, что

$$
\begin{aligned}
& \frac{1}{N} \sum_{k=1}^{N} a_{k}(x) \rightarrow a(x) \quad \mu \text {-почти всюду, } \\
& \frac{1}{N} \sum_{k=1}^{N} b_{k}(y) \rightarrow b(y) \quad \nu \text {-почти всюду }
\end{aligned}
$$

для некоторых неотрицательных функций $a \in L^{1}(X), b \in L^{1}(Y)$. Рассмотрим функцию $g(x, y):=a(x)+b(y)$. Тогда

$$
\frac{1}{N} \sum_{k=1}^{N} g_{n}(x, y) \rightarrow g(x, y) \quad(\mu \times \nu) \text {-почти всюду. }
$$

Ясно, что в этом случае $g \in B$. С другой стороны,

$$
\int a+\int b \leqslant \limsup _{N} \frac{1}{N} \sum_{k=1}^{N}\left(\int a_{k}+\int b_{k}\right) \leqslant\|f\|_{\mathrm{SR}^{1}}-\theta
$$

в силу полунепрерывности снизу интеграла неотрицательных функций относительно сходимости почти всюду. Таким образом, $g \in A$. Противоречие.

Воспользуемся теперь теоремой отделимости Хана-Банаха. Так как множество $A$ содержит 0, найдется функция $h \in L^{\infty}(X \times Y)$ такая, что $\int g h<1$ для любой $g \in A$ и $\int g h>1$ для любой $g \in B$. Так как множество $B$ является сдвигом конуса неотрицательных функций и $\int g h>1$ для любой $g \in B$, мы получаем, что $h \geqslant 0$ почти всюду. Для произвольного множества $X_{1} \subset X$ возьмем функцию

$$
a(x)=\frac{\|f\|_{\mathrm{SR}^{1}}-\theta}{\mu\left(X_{1}\right)} \chi_{X_{1}}(x) .
$$

Тогда $a(x) \in A$, поэтому

$$
\frac{1}{\mu\left(X_{1}\right)} \int_{X_{1}} \int_{Y}\left(\|f\|_{\mathrm{SR}^{1}}-\theta\right) h(x, y) d \nu(y) d \mu(x) \leqslant 1 .
$$

Аналогичные неравенства можно написать, поменяв переменные, поэтому можно заключить, что функция $\widetilde{h}=\left(\|f\|_{\mathrm{SR}^{1}}-\theta\right) h$ принадлежит множеству $\mathscr{S}$. Но $|f| \in B$, поэтому

$$
\int|f| \widetilde{h}=\left(\|f\|_{\mathrm{SR}^{1}}-\theta\right) \int|f| h \geqslant\|f\|_{\mathrm{SR}^{1}}-\theta .
$$

В силу произвольности $\theta$, теорема 8 доказана.

Докажем теперь еще одну теорему о введенной $\mathrm{SR}^{1}$-норме.

Теорема 9. Для любой измеримой функиии $f: X \times Y \rightarrow \mathbb{R}$ выполнены неравенства

$$
\frac{1}{4}\|f\|_{\mathrm{SR}^{1}} \leqslant \int_{0}^{\infty} \operatorname{th}\{|f| \geqslant \lambda\} d \lambda \leqslant 2\|f\|_{\mathrm{SR}^{1}} .
$$


ДокАЗАТЕЛЬСтво. Если $|f(x, y)| \stackrel{\bmod 0}{\leqslant} a(x)+b(y)$, то

$\{(x, y):|f(x, y)| \geqslant \lambda\} \stackrel{\bmod 0}{\subset}\left(\left\{x: a(x) \geqslant \frac{\lambda}{2}\right\} \times Y\right) \cup\left(X \times\left\{y: b(y) \geqslant \frac{\lambda}{2}\right\}\right)$,

поэтому

$$
\operatorname{th}\{|f| \geqslant \lambda\} \leqslant \mu\left\{a \geqslant \frac{\lambda}{2}\right\}+\nu\left\{b \geqslant \frac{\lambda}{2}\right\} .
$$

Интегрируя это неравенство по $\lambda$, получаем

$$
\int_{0}^{\infty} \operatorname{th}\{|f| \geqslant \lambda\} d \lambda \leqslant \int_{0}^{\infty}\left(\mu\left\{a \geqslant \frac{\lambda}{2}\right\}+\nu\left\{b \geqslant \frac{\lambda}{2}\right\}\right) d \lambda=2\left(\int_{X} a+\int_{Y} b\right) .
$$

Переходя к инфимуму по парам функций $a$ и $b$, получаем правое неравенство в (6).

Докажем теперь левое неравенство. Так как $\operatorname{th}\{|f| \geqslant \lambda\}$ монотонно убывает по $\lambda$, то выполнено такое неравенство:

$$
\int_{0}^{\infty} \operatorname{th}\{|f| \geqslant \lambda\} d \lambda \geqslant \sum_{k \in \mathbb{Z}} 2^{k-1} \operatorname{th}\left\{|f| \geqslant 2^{k}\right\} .
$$

Для произвольного $\varepsilon>0$ выберем множества $A_{k}$ и $B_{k}$ так, что

$$
\left\{|f| \geqslant 2^{k}\right\} \stackrel{\bmod 0}{\subset}\left(A_{k} \times Y\right) \cup\left(X \times B_{k}\right)
$$

и

$$
\mu\left(A_{k}\right)+\nu\left(B_{k}\right) \leqslant(1+\varepsilon) \operatorname{th}\left\{|f| \geqslant 2^{k}\right\} .
$$

Возьмем функции $a(x)=\sum 2^{k+1} \chi_{A_{k}}(x)$ и $b(y)=\sum 2^{k+1} \chi_{B_{k}}(y)$. Легко проверить, что $|f(x, y)| \stackrel{\bmod 0}{\leqslant} a(x)+b(y)$, поэтому

$$
\|f\|_{\mathrm{SR}^{1}} \leqslant \int_{X} a+\int_{Y} b \leqslant 4(1+\varepsilon) \sum_{k \in \mathbb{Z}} 2^{k-1} \operatorname{th}\left\{|f| \geqslant 2^{k}\right\} .
$$

Последнее неравенство вместе с (7), в силу произвольности $\varepsilon$, доказывает требуемое. Теорема доказана.

Из этой теоремы получаем полезное следствие.

СледСтвиЕ 4. Если $\|f\|_{\mathrm{SR}^{1}}<\infty$, то функиия $f$ приближается по $\mathrm{SR}^{1}$-норме своими срезками.

ДоказАтельство. Пусть $f_{N}$ - двусторонняя срезка $f$ по уровню $N$. Тогда $\left\{\left|f-f_{N}\right| \geqslant \lambda\right\} \subset\{|f| \geqslant N+\lambda\}$, поэтому

$$
\int_{0}^{\infty} \operatorname{th}\left\{\left|f-f_{N}\right| \geqslant \lambda\right\} d \lambda \leqslant \int_{0}^{\infty} \operatorname{th}\{|f| \geqslant N+\lambda\} d \lambda=\int_{N}^{\infty} \operatorname{th}\{|f| \geqslant \lambda\} d \lambda \rightarrow 0
$$

при $N \rightarrow \infty$. По теореме $9,\left\|f-f_{N}\right\|_{\mathrm{SR}^{1}} \rightarrow 0$. Следствие доказано.

ТеОрема 10. Замыкание множества ступенчатых функиици в $\mathrm{SR}^{1}$-норме состоит из всех виртуально непрерывных функицй, у которых эта норма конечна (в частности, любая ограниченная виртуалъно непрерывная функиия лежит в этом замыкании). 
ДоКАЗАТЕЛЬСтво. Для того чтобы доказать, что предел по норме последовательности ступенчатых функций является виртуально непрерывной функцией, достаточно воспользоваться следствием 3 и теоремой 6 .

Докажем теперь, что каждая виртуально непрерывная функция с конечной $\mathrm{SR}^{1}$-нормой приближается по этой норме ступенчатыми функциями. Пусть $\|f\|_{\mathrm{SR}^{1}}<\infty$ и $\varepsilon>0$. По следствию 4 , при достаточно большом $N$ двусторонняя срезка $f_{N}$ функции $f$ по уровню $N$ хорошо приближает функцию $f$ по норме: $\left\|f-f_{N}\right\|_{\mathrm{SR}^{1}}<\varepsilon$. Зафиксируем такое большое $N$. Далее, функция $f_{N}$, очевидно, является виртуально непрерывной, поэтому по теореме 6 она приближается в метрике $\tau$ ступенчатыми функциями, т. е. найдется ступенчатая функция $g$ такая, что $\tau\left(g, f_{N}\right)<\varepsilon / N$. При этом функцию $g$ можно считать ограниченной по модулю той же константой $N$ (если это не так, ее можно срезать). Так как $\tau\left(g, f_{N}\right)<\varepsilon / N$, найдутся множества $X_{0} \subset X, Y_{0} \subset Y$ такие, что $\mu\left(X_{0}\right)<\varepsilon / N$, $\nu\left(Y_{0}\right)<\varepsilon / N$ и $\left|f_{N}-g\right|<\varepsilon / N$ почти всюду на $\left(X \backslash X_{0}\right) \times\left(Y \backslash Y_{0}\right)$. Но тогда мы имеем неравенство

$$
\left|f_{N}(x, y)-g(x, y)\right| \stackrel{\bmod 0}{\leqslant} \frac{\varepsilon}{N}+2 N \chi_{X_{0}}(x)+2 N \chi_{Y_{0}}(y)
$$

поэтому

$$
\left\|f_{N}-g\right\|_{\mathrm{SR}^{1}} \leqslant \frac{\varepsilon}{N}+2 N\left(\mu\left(X_{0}\right)+\nu\left(Y_{0}\right)\right) \leqslant 5 \varepsilon .
$$

Значит, $\|f-g\|_{\mathrm{SR}^{1}} \leqslant 6 \varepsilon$. Теорема доказана.

Пространство всех виртуально непрерывных функций с конечной $\mathrm{SR}^{1}$-нормой обозначим $V C^{1}$. Оно является аналогом пространства $L^{1}$ для виртуально непрерывных функций и является предсопряженным к пространству полиморфизмов с ограниченными плотностями проекций.

ТеОрема 11. Сопряженным $\kappa$ пространству $V C^{1}$ является пространство $Q B^{\infty}$ квазибистохастических зарядов $\eta$ на $X \times Y$ с конечной нормой

$$
\|\eta\|_{\mathrm{qb}}=\max \left\{\left\|\frac{\partial P_{*}^{x}|\eta|}{\partial \mu}\right\|_{L^{\infty}(X, \mu)},\left\|\frac{\partial P_{*}^{y}|\eta|}{\partial \nu}\right\|_{L^{\infty}(Y, \nu)}\right\},
$$

где $P^{x}$ и $P^{y}$ - проекиии на $X$ и $Y$ соответственно, а $|\eta|$ - вариация заряда. Действие заряда $\eta$ на виртуально непрерьвную функиию $f$ определяется $к а к \int \tilde{f} d \eta$, где $\widetilde{f}$ - собственно виртуально непрерывная функция, эквивалентная $f .1$

Для доказательства теоремы 11 нам понадобится следующая лемма.

Лемма 9. Пусть $K$-метрический компакт, $F$ - непрерывный линейный функционал на пространстве непрерывных функиий $C(K)$. Непрерывные на $K$ функции $f_{1}, f_{2}, \ldots$ таковы, что их нормы равномерно ограничены, а носители не пересекаются. Тогда ряд $\sum F\left(f_{i}\right)$ сходится абсолютно. Если при этом (заданная поточечно) функция $f=\sum f_{i}$ непрерывна, то $F(f)=\sum F\left(f_{i}\right)$.

\footnotetext{
${ }^{1}$ Согласно замечанию в конце п. 2.6, функция $\widetilde{f}$ будет $\eta$-измеримой и значение интеграла не зависит от выбора $\widetilde{f}$.
} 
ДокАзАтельство. Функционал $F$ по теореме Рисса задается интегрированием по борелевской мере со знаком, имеющей конечную вариацию. Абсолютная сходимость ряда следует из счетной аддитивности этой меры и конечности вариации. Искомое равенство $F(f)=\sum F\left(f_{i}\right)$ следует из теоремы Лебега о суммируемой мажоранте. Лемма доказана.

ДокАЗАТЕЛЬСТво ТЕОРЕмы 11. Пусть сначала $\eta$ - такая знакопеременная мера, что $\|\eta\|_{\text {qb }}<\infty$. Заметим, что если для ступенчатой функции $h$ оценка $|h(x, y)| \leqslant a(x)+b(y)$ имеет место почти всюду, то она имеет место и почти всюду на произведении множеств полной меры, стало быть, $|\eta|$-почти всюду. Поэтому такое неравенство можно интегрировать по $|\eta|$, откуда

$$
\begin{aligned}
\left|\int h(x, y) d \eta\right| & \leqslant \int|h| d|\eta| \leqslant \int(a(x)+b(y)) d|\eta| \\
& \leqslant\|\eta\|_{\mathrm{qb}}\left(\int|a(x)| d \mu(x)+\int|b(y)| d \nu(y)\right) .
\end{aligned}
$$

Переходя к инфимуму по $a, b$ таким, что $|h| \stackrel{\bmod 0}{\leqslant} a(x)+b(y)$, получаем неравенство $\left|\int h d \eta\right| \leqslant\|h\|_{\mathrm{SR}^{1}}\|\eta\|_{\mathrm{qb}}$, что и требовалось.

Теперь покажем, что любой линейный непрерывный функционал $F$ на пространстве $V C^{1}$ задается указанным образом. Не умаляя общности, можно считать, что $\|F\|=1$. Для ступенчатого множества (т. е. конечного объединения прямоугольников) $Z \subset X \times Y$ положим

$$
\begin{aligned}
\eta(Z) & :=F\left(\chi_{Z}\right), \\
|\eta|(Z) & :=\sup \sum_{\sqcup Z_{i} \subset Z}\left|\eta\left(Z_{i}\right)\right|,
\end{aligned}
$$

где супремум берется по всем последовательностям непересекающихся ступенчатых множеств $Z_{1}, Z_{2}, \ldots$ в $Z$. Очевидно, что супремум можно брать лишь по конечным наборам, более того, в качестве $Z_{i}$ достаточно брать лишь прямоугольники. Введенные функции множества конечно аддитивны.

Для любого конечного набора непересекающихся ступенчатых множеств $Z_{i} \subset Z$ имеем $\sum\left|\eta\left(Z_{i}\right)\right|=F\left(\sum\left( \pm \chi_{Z_{i}}\right)\right)$. Кроме того, $\left| \pm \chi_{Z_{i}}\right| \leqslant \chi_{Z}$. Если $Z=X_{1} \times Y$, то $\left\|\sum\left( \pm \chi_{Z_{i}}\right)\right\|_{\mathrm{SR}^{1}} \leqslant \mu\left(X_{1}\right)$, поэтому $|\eta|\left(X_{1} \times Y\right) \leqslant \mu\left(X_{1}\right)$. Аналогично, $|\eta|\left(X \times Y_{1}\right) \leqslant \nu\left(Y_{1}\right)$. Докажем, что конечно аддитивные функции множества $\eta$ и $|\eta|$, заданные на алгебре ступенчатых множеств, продолжаются до меры со знаком и меры на всей $\sigma$-алгебре $\mathfrak{A} \times \mathfrak{B}$ пространства $X \times Y$.

Для этого, согласно теореме Колмогорова-Хана, достаточно проверить, что если $Z_{i}$ - непересекающиеся ступенчатые множества и множество $Z=\bigsqcup Z_{i}$ тоже ступенчатое, то $|\eta|(Z)=\sum|\eta|\left(Z_{i}\right)$. Так как $|\eta|$ - предмера, то неравенство $|\eta|(Z) \geqslant \sum|\eta|\left(Z_{i}\right)$ очевидно. Осталось доказать неравенство в другую сторону. По определению, величина $|\eta|(Z)$ есть супремум сумм $\sum\left|\eta\left(P_{k}\right)\right|$ по всем конечным наборам непересекающихся прямоугольников $P_{k}$, содержащихся в $Z$, поэтому достаточно доказать, что

$$
\sum_{k}\left|\eta\left(P_{k}\right)\right| \leqslant \sum_{i}|\eta|\left(Z_{i}\right)
$$


В силу конечной аддитивности предмеры $|\eta|$ достаточно доказать, что для каждого прямоугольника $P_{k}$ выполнено неравенство

$$
\left|\eta\left(P_{k}\right)\right| \leqslant \sum_{i}|\eta|\left(Z_{i} \cap P_{k}\right) .
$$

Разбив каждое из множеств $Z_{i} \cap P_{k}$ на конечное количество прямоугольников, мы сводим это к неравенству такого вида:

$$
|\eta(Q)| \leqslant \sum_{i}\left|\eta\left(Q_{i}\right)\right|
$$

где прямоугольник $Q$ есть объединение непересекающихся прямоугольников $Q_{i}$.

Рассматривая ряд из разрезных полуметрик, несложно построить такие допустимые полуметрики $\rho_{X}, \rho_{Y}$, что метрические пространства $\left(X, \rho_{X}\right),\left(Y, \rho_{Y}\right)$ будут предкомпактны, а проекция каждой стороны каждого из прямоугольников $Q_{i}$ и $Q$ будет находиться на положительном расстоянии от своего дополнения. Тогда все функции $\chi_{Q_{i}}$ и $\chi_{Q}$ являются равномерно непрерывными на пространстве $\left(X \times Y, \rho_{X} \times \rho_{Y}\right)$ и поэтому продолжаются непрерывно на пополнение (единицей на замыкание соответствующего прямоугольника и нулем на замыкание его дополнения). При этом носители продолженных функций по-прежнему не пересекаются. Пространство непрерывных функций на пополнении $X \times Y$ вкладывается в $V C^{1}$ с нормой вложения не более 1 , так что функционал $F$ является на нем непрерывным. Применив лемму 9 к последовательности функций $\chi_{Q_{i}}$, получаем равенство

$$
\eta(Q)=F\left(\chi_{Q}\right)=\sum_{i} F\left(\chi_{Q_{i}}\right)=\sum_{i} \eta\left(Q_{i}\right)
$$

из которого следует искомое неравенство. Теорема 11 доказана.

СЛЕДСТВИЕ 5. Для виртуально непреръвнъх функиий из пространства $V C^{1}$ (в частности, для ограниченных виртуально непрерывных функций) корректно определен интеграл не только по множествам положительной меры, как для произвольных суммируемых функций, но и интеграл по бистохастическим (сингулярным) мерам, например, по мере Лебега на диагонали $\{x=y\} \subset[0,1]^{2}$ или по мере, сосредоточенной на графике преобразования с квазиинвариантой мерой. Таким образом, виртуально непрерывные функици имеют "след (ограничение) на диагонали" в смысле теорем о следах.

В качестве приложения докажем следующий вариант непрерывной теоремы Холла, борелевская версия которого приведена в приложениях [8] к книге Л. Ловаса [7].

Теорема 12. Пусть $Z \subset X \times Y$ - виртуально замкнутое множество. Тогда равносильны следующие два условия:

(i) найдется бистохастическая мера $\lambda$ такая, что $\lambda(Z)=1$;

(ii) собственная толщина $\operatorname{sth}(Z)$ множества $Z$ равна 1.

Доказательство. Условие (i) непосредственно влечет (ii). Докажем обратную импликацию. Можно считать, что множество $Z$ замкнуто по метрике $d=\rho_{X} \times \rho_{Y}$, где $\rho_{X}, \rho_{Y}-$ допустимые метрики на $X, Y$. Тогда функция $f(x, y):=\exp \{-d((x, y), Z)\}$ будет собственно виртуально непрерывна. 
Докажем, что ее $V C^{1}$-норма равна 1. Если это не так, то найдутся неотрицательные функции $a(x), b(y)$ такие, что $a(x)+b(y) \geqslant f(x, y)$ почти всюду и

$$
\int a+\int b=e^{-2 \varepsilon}, \quad \varepsilon>0 .
$$

Рассмотрим $\varepsilon$-окрестность $Z_{\varepsilon}$ множества $Z$ в метрике $d$. Это множество виртуально открыто, так что по лемме 3 для него собственная толщина совпадает с толщиной (и потому равна 1). Имеем

$$
a(x)+b(y) \geqslant e^{-\varepsilon} \chi_{Z_{\varepsilon}}
$$

при почти всех $x \in X, y \in Y$, так что

$$
\operatorname{th}\left(Z_{\varepsilon}\right) \leqslant e^{\varepsilon}\left(\int a+\int b\right)=e^{-\varepsilon} .
$$

Противоречие.

Для элемента с единичной нормой в банаховом пространстве найдется непрерывный функционал единичной нормы, принимающий на этом элементе значение 1. По теореме 11 этот функционал задается суббистохастическим зарядом $\lambda$ и $\int f d \lambda=1$. Но

$$
\left|\int f d \lambda\right| \leqslant \int|f| d|\lambda|=\int_{0}^{1}|\lambda|\left(f^{-1}[t, 1]\right) d t \leqslant 1,
$$

и, поскольку все неравенства обращаются в равенства, $\lambda(Z)=\lambda\left(f^{-1}\{1\}\right)=1$. Теорема доказана.

Отсюда несложно вывести следующее утверждение.

СлЕДСТвИЕ 6. Для виртуально замкнутого множества $Z$ всегда имеет место равенство $\max \lambda(Z)=\operatorname{sth}(Z)$, где максимум берется по всем бистохастическим мерам $\lambda$.

ДокАЗАТЕЛЬство. Применим стандартный трюк: добавим к пространствам $X$ и $Y$ пространства $X_{0}, Y_{0}$ меры $1-\operatorname{sth}(Z)$ соответственно, отнормируем меры на $X \sqcup X_{0}$ и $Y \sqcup Y_{0}$. Рассмотрим в $\left(X \sqcup X_{0}\right) \times\left(Y \sqcup Y_{0}\right)$ множество $Z \sqcup X_{0} \times$ $Y_{0} \sqcup X_{0} \times Y \sqcup X \times Y_{0}$ собственной толщины 1. Для этого множества используем теорему 12 ; часть соответствующей бистохастической меры, лежащая в $X \times Y$, будет искомой. Следствие доказано.

\section{3. Приложения: транспортная задача, теоремы вложения, теоремы о следах и ограничение метрик}

Укажем на некоторые приложения понятия виртуальной непрерывности.

3.1. Двойственность Канторовича в транспортной задаче. Свяжем теорему 11 о пространстве квазиполиморфизмов $Q B^{\infty}$, сопряженном пространству виртуально непрерывных функций $V C^{1}$, с классической теоремой Л. В. Канторовича о двойственности в непрерывной линейной транспортной задаче. Напомним ее. 
Рассматривается метрическое пространство ${ }^{2}(X, \rho)$ и две вероятностные борелевские меры $\mu_{1}, \mu_{2}$ на нем. Ищется

$$
\inf \left\{\int_{X} \int_{X} \rho(x, y) d \Psi(x, y): \Psi \in Q B S_{\mu_{1}, \mu_{2}}^{\infty}\right\},
$$

где $Q B S_{\mu_{1}, \mu_{2}}^{\infty}-$ совокупность мер на $X \times X$ с проекциями (маргинальными) на координаты, равными мерам $\mu_{1}, \mu_{2}$ (другие названия таких мер $\Psi$ - полиморфизм $\left(X, \mu_{1}\right)$ в $\left(X, \mu_{2}\right)$, план перевозок, каплинг, стыковка, мера Юнга и т. д.).

Основные факты, объединенные названием "теорема двойственности", изложенные в наших терминах, состоят в том, что:

$1)$ инфимум (8) достигается на некотором неотрицательном элементе $\Psi_{0}$ пространства $Q B S_{\mu_{1}, \mu_{2}}^{\infty}$ (и не достигается, вообе говоря, на пространстве абсолютно непрерывных мер вида $d \Psi=p(x, y) d \mu_{1}(x) d \mu_{2}(y)$, где $p$ - измеримая суммируемая функция);

2) этот инфимум можно рассматривать как норму знакопеременной меры $\left\|\mu_{1}-\mu_{2}\right\|$ в некотором пространстве знакопеременных борелевских мер на пространстве $(X, \rho)$ с конечной вариацией мер (это наблюдение, сделанное в работе [5], положило начало традиции называть эту норму нормой Канторовича-Рубинштейна, а метрику - метрикой Канторовича);

3) имеет место двойственное определение нормы

$$
\left\|\mu_{1}-\mu_{2}\right\|=\sup \left\{\int_{X} u(x) d\left(\mu_{1}-\mu_{2}\right)(x): u \in \operatorname{Lip}_{1}(\rho)\right\},
$$

где $\operatorname{Lip}_{1}(\rho)$ - единичный шар в пространстве липшицевых функций с обычной нормой, при этом супремум тоже достигается на некоторой липшицевой функции $u_{0}$ и имеет место равенство $u(x)-u(y)=\rho(x, y)$ почти всюду по мере $\Psi_{0}$.

Главное содержание этих утверждений в том, что норму элемента банахова пространства можно вычислять с помощью функционалов из сопряженного пространства, что сводит дело к поиску сопряженного пространства.

Приведенное утверждение называется теоремой двойственности (или критерием оптимальности) в транспортной задаче и было сформулировано в пионерской работе [4]. Фактически, используется лишь то, что пространство липшицевых функций есть банахово сопряженное к пространству мер с нормой Канторовича-Рубинштейна. Полезно подчеркнуть, что эта теорема - о функциях и мерах "одного аргумента", и мы дополняем (точнее, накрываем) ее теоремой двойственности для функций двух аргументов.

Ниже мы покажем, как из доказанной выше теоремы 11, которая тоже есть теорема о сопряженном пространстве к пространству $V C^{1}$ виртуально непрерывных функций двух аргументов, можно вывести теорему двойственности Канторовича. Более точно, мы формулируем утверждение о естественном "накрытии" этой теоремы более общей теоремой двойственности для пространств функций двух аргументов. При этом нам будет удобнее говорить о перевозке массы не в пределах одного пространства, а из одного пространства в другое (разумеется, эти задачи эквивалентны).

\footnotetext{
${ }^{2}$ В классической работе [4] это компакт, но для дальнейшего важно лишь, что это сепарабельное полное метрическое (= польское) пространство.
} 
Тем самым, мы получаем еще одно доказательство теоремы двойственности, но главное состоит в том, что наша схема включает в рассмотрение пространства метрик и пространства планов, чего нет в схеме Канторовича. Естественность выбора пространств $V C^{1}$ и $Q B^{\infty}$ в том, что нельзя обойтись меньшими пространствами планов (см. замечание выше), и в том, что допустимые метрики являются виртуально непрерывными функциями.

В свою очередь, наблюдение о двухуровневой теореме двойственности в нашей конкретной ситуации позволяет сформулировать общую двухуровневую теорему двойственности, которая, как мы надеемся, найдет и другие применения. Поэтому мы сначала формулируем общее утверждение, а затем показываем, как оно применяется к рассматриваемому случаю.

Теорема 13. Пусть $X$ - вещественное векторное пространство, упорядоченное выпуклым конусом $K, u Y=X^{*}$ - сопряженное пространство. Пусть $W$ и $Z=W^{*}-$ два других линейных вещественных пространства u рассматриваются оператор $A: W \rightarrow X$ u сопряюсенный оператор $B=$ $A^{*}: Y \rightarrow Z:$

$$
W \stackrel{A}{\longrightarrow} X, \quad X^{*} \stackrel{A^{*}}{\longrightarrow} W^{*} .
$$

Зафиксируем положительный элемент $\rho \in K \subset X$ и определим (конечную или бесконечную) квазинорму на $Z$ формулой

$$
\|z\|_{\rho}=\inf \{(y, \rho): B y=z, y \geqslant 0\} .
$$

Предположим, что выполняется следующее условие: пространство X есть сумма конуса $K$ и пространства $A(W)$. Тогда

$$
\|z\|_{\rho}=\|z\|_{\rho}^{\prime}:=\sup \{(z, u): A u \leqslant \rho\} .
$$

Кроме того, если $\|z\|_{\rho}<\infty$, то найдется неотрицательный непрерывный бункиионал $y \in X^{*}$ такой, что $(y, \rho)=\|z\|_{\rho}$.

ЗАмечАниЕ 1 . В случае, когда $X$ - банахово пространство, а конус $K$ - замкнутый и порождающий $(K-K=X)$, в силу классической теоремы Какутани неотрицательный функционал $y$ на $X$ автоматически оказывается ограниченным по норме в $X$.

ДокАЗАТЕЛЬство. Ясно, что всегда $\|z\|_{\rho}^{\prime} \leqslant\|z\|_{\rho}$. Действительно, фиксируя элемент $y \in Y$ такой, что $y \geqslant 0$ и $B y=z$, и элемент $u \in W$ такой, что $A u \leqslant \rho$, имеем

$$
(z, u)=(B y, u)=(y, A u)=(y, \rho)-(y, \rho-A u) \leqslant(y, \rho) ;
$$

равенство достигается, если $(y, \rho-A u)=0$.

Предположим, что

$$
C:=\|z\|_{\rho}^{\prime}=\sup \{(z, u): A u \leqslant \rho\}<\|z\|_{\rho} .
$$

Заметим, что из конечности величины $C$ следует, что $(z, w)=0$ при $A w=0$ (иначе рассмотрим $u=\lambda w$ при вещественном $\lambda$ подходящего знака, спаривание $(z, u)$ будет неограничено). Это означает, что $z \in B(Y)$, поскольку образ сопряженного оператора есть аннулятор ядра прямого. Кроме того, если $A w \geqslant 0$, то $(z, w) \geqslant 0$, иначе рассмотрим $u=\lambda w$ при отрицательном $\lambda$. Если $\rho=A u_{0}$ при некотором $u$, то для любого $y$ такого, что $B y=z$, имеем

$$
\left(z, u_{0}\right)=\left(B y, u_{0}\right)=\left(y, A u_{0}\right)=(y, \rho),
$$


что и требовалось. Пусть $\rho \notin A(W)$. Будем искать функционал $y \in Y=X^{*}$ такой, что $(y, A u)=(z, u)$ при всех $u \in W$ (это как раз означает, что $z=A^{*} y=$ $B y), y \geqslant 0,(y, \rho)=C$. На линейной оболочке пространства $A(W)$ и элемента $\rho$ функционал $y$ уже задан, и на ней он неотрицателен. Последнее утверждение верно для неотрицательных элементов вида $\rho-A u$ по определению числа $C$, так что нужно проверить его еще на неотрицательных элементах вида $A u-\rho$. Пусть $\varepsilon>0$, тогда найдется элемент $w \in W$ такой, что $A w \leqslant \rho$ и $(y, A w)=$ $(z, w) \geqslant C-\varepsilon$. Имеем

$$
(y, A u-\rho)=(y, A u)-C \geqslant(y, A u)-(y, A w)-\varepsilon=(y, A(u-w))-\varepsilon \geqslant-\varepsilon,
$$

поскольку $A(u-w)=(A u-\rho)+(\rho-A w) \geqslant 0$, а на образе $A(W)$ функционал $y$ неотрицателен. В силу произвольности $\varepsilon>0$ имеем $(y, A u-\rho) \geqslant 0$.

Пространство $A(W)$ и конус $K$ порождают $X$, по теореме Рисса о продолжении неотрицательного функционала $y$ можно продолжить до неотрицательного функционала, заданного на всем пространстве $X$. Теорема доказана.

В интересующей нас ситуации $X=V C^{1}\left(\Omega_{1} \times \Omega_{2}\right), Y \supset Q B^{\infty}\left(\Omega_{1} \times \Omega_{2}\right)$, где $\left(\Omega_{i}, \mu_{i}\right), i=1,2,-$ вероятностные пространства Лебега, $W=L^{1}\left(\Omega_{1}\right) \oplus$ $L^{1}\left(\Omega_{2}\right), Z=L^{\infty}\left(\Omega_{1}\right) \oplus L^{\infty}\left(\Omega_{2}\right)$, оператор $A$ отображает пару функций $u=$ $\left(w_{1}(t), w_{2}(t)\right) \in W$ в функцию $w_{1}\left(t_{1}\right)+w_{2}\left(t_{2}\right):=(A u)\left(t_{1}, t_{2}\right) \in X$, сужение $B$ на $Q B^{\infty}$ отображает квазибистохастический заряд $\eta$ в пару его проекций на $X$ и на $Y$ :

$$
\begin{aligned}
L^{1}\left(\Omega_{1}\right) \times L^{1}\left(\Omega_{2}\right) \stackrel{A}{\longrightarrow} V C^{1}\left(\Omega_{1}, \Omega_{2}\right), \\
\left(w_{1}(x), w_{2}(y)\right) \stackrel{A}{\longmapsto} w_{1}(x)+w_{2}(y) ; \\
Q B^{\infty}\left(\Omega_{1}, \Omega_{2}\right) \stackrel{A^{*}}{\longmapsto} L^{\infty}\left(\Omega_{1}\right) \times L^{\infty}\left(\Omega_{2}\right), \\
\mu \stackrel{A^{*}}{\longmapsto}\left(\operatorname{Pr}_{\Omega_{1}} \mu, \operatorname{Pr}_{\Omega_{2}} \mu\right) .
\end{aligned}
$$

Элемент $\rho\left(t_{1}, t_{2}\right)$ будем понимать как функцию цены перевозки из точки $t_{1} \in \Omega_{1}$ в точку $t_{2} \in \Omega_{2}$, в качестве элемента $z \in Z$ выберем пару постоянных функций $(1,1)$ (это не ограничивает общность: для других функций можно просто поменять меры $\mu_{1}, \mu_{2}$ на эквивалентные). Заметим, что по определению пространства $V C^{1}$ всякая функция в нем представима в виде суммы неотрицательной функции и функции вида $w_{1}\left(t_{1}\right)+w_{2}\left(t_{2}\right)$. Таким образом, условие теоремы 13 выполняется. В силу замечания 1 к теореме 13 (очевидно, что конус неотрицательных функций в $V C^{1}$ замкнутый и порождающий) этот функционал задается некоторым полиморфизмом из $Q B^{\infty}$. Норма $\|(1,1)\|_{\rho}$ равна инфимуму стоимостей планов переноса меры $\mu_{1}$ в меру $\mu_{2}$ с ценовой функцией $\rho$. Таким образом, из теоремы 13 следует, что оптимальный план в транспортной задаче существует.

Примером ситуации, когда не выполнены ни условие, ни заключение теоремы 13, может служить аналог предыдущего примера, в котором вместо пространства $V C^{1}\left(\Omega_{1} \times \Omega_{2}\right)$ рассматривается $L^{1}\left(\Omega_{1} \times \Omega_{2}\right)$. В этом случае неотрицательные ограниченные функционалы задаются неотрицательными ограниченными функциями (а не полиморфизмами) и оптимальный план вполне может не существовать. 


\section{2. Пространства Соболева и теоремы о следах.}

ТЕОРема 14. Пусть $\Omega_{1}, \Omega_{2}$ - области размерностей $d_{1}, d_{2}$ соответственно, $p l>d_{2}$ либо $p=1, l=d_{2}$. Тогда функции из $W_{p}^{l}\left(\Omega_{1} \times \Omega_{2}\right)$ (l-е обобщенные производные суммируемы со степенью р) виртуально непрерывны как функиии двух переменных $x \in \Omega_{1}, y \in \Omega_{2}$. Вложение $W_{p}^{l}\left(\Omega_{1} \times \Omega_{2}\right)$ в $V C^{1}\left(\Omega_{1}, K\right)$ непрерывно для любого компакта $K$ в области $\Omega_{2}$.

ДокАЗАТЕЛЬство. Согласно теореме вложения в непрерывные функции (см., например, [1], [9]), для функций $h(y) \in W_{p}^{l}\left(\Omega_{2}\right)$ имеет место оценка

$$
\|h\|_{C(K)} \leqslant c\left(\Omega_{2}, K\right)\|h\|_{W_{p}^{l}\left(\Omega_{2}\right)} .
$$

Пусть $f(x, y) \in W_{p}^{l}\left(\Omega_{1} \times \Omega_{2}\right)$ - гладкая функция. Положим

$$
a(x):=\|f(x, \cdot)\|_{W_{p}^{l}\left(\Omega_{2}\right)} .
$$

Тогда по теореме Фубини $a \in L^{1}\left(\Omega_{1}\right)$ и

$$
\int|a| \leqslant c\left(\Omega_{1}, \Omega_{2}\right)\|f\|_{W_{p}^{l}\left(\Omega_{1} \times \Omega_{2}\right)} .
$$

На $\Omega_{1} \times K$ имеем

$$
|f(x, y)| \leqslant\|f(x, \cdot)\|_{C(K)} \leqslant c\left(\Omega_{2}, K\right) a(x) .
$$

Резюмируя, получаем, что

$$
\|f\|_{V C^{1}\left(\Omega_{1}, K\right)} \leqslant c\left(\Omega_{1}, \Omega_{2}, K\right)\|f\|_{W_{p}^{l}\left(\Omega_{1} \times \Omega_{2}\right)} .
$$

Любая функция из класса $W_{p}^{l}\left(\Omega_{1} \times \Omega_{2}\right)$ есть предел последовательности гладких функций, который в силу (9) является и пределом в $V C^{1}$. Теорема доказана.

Таким образом, в условиях указанной теоремы о вложении мы получаем возможность интегрировать функции по любой квазибистохастической мере, что обобщает обычные теоремы о следах на подмногообразиях.

3.3. Ядерные операторы в гильбертовом пространстве. Хорошо известно, что пространство ядерных операторов в гильбертовом пространстве $L^{2}$ есть проективное тензорное произведение гильбертовых пространств. Их ядра суть измеримые функции двух переменных, прямое описание которых труднообозримо. Следующая теорема утверждает, что ядра ядерных интегральных операторов как функции двух переменных виртуально непрерывны. Заметим, что ядра операторов Гильберта-Шмидта, вообе говоря, не являются виртуально непрерывными.

Теорема 15. Пусть $(X, \mu),(Y, \nu)$ - стандартные вероятностные пространства. Пространство ядер ядерных операторов из $L^{2}(X)$ в $L^{2}(Y)$ (с нормой Шаттена-фон Неймана) непрерывно вкладывается в $\mathrm{VC}^{1}$.

ДокАЗАТЕЛЬСтво. Пусть $K(x, y)$ - ядро интегрального оператора конечного ранга из $L^{2}(X)$ в $L^{2}(Y)$ с ядерной нормой 1 . Тогда имеет место разложение в конечную сумму

$$
K(x, y)=\sum s_{k} a_{k}(x) b_{k}(y)
$$


где $s_{k}$ - сингулярные числа оператора, $\left(a_{k}\right),\left(b_{k}\right)$ - ортонормированные системы, $\sum\left|s_{k}\right| \leqslant 1$. Почти всюду имеем

$$
|K(x, y)| \leqslant \frac{1}{2} \sum\left|s_{k}\right|\left|a_{k}^{2}(x)\right|+\frac{1}{2} \sum\left|s_{k}\right|\left|b_{k}^{2}(y)\right|
$$

Правая часть неравенства имеет вид $A(x)+B(y)$, причем

$$
\int|A(x)| d x+\int|B(y)| d y \leqslant 1 .
$$

Таким образом, норма функции $K(x, y)$ в пространстве $V C^{1}$ не превосходит 1. Осталось заметить, что любой ядерный оператор аппроксимируется по ядерной норме операторами конечного ранга, и, по доказанному, эта аппроксимация будет аппроксимацией и в $V C^{1}$. Теорема доказана.

Отсюда следует, что эти ядра можно интегрировать не только по диагонали, что хорошо известно, но и по бистохастическим мерам. Однако пространство $V C^{1}$ не исчерпывается этими ядрами. Следует также помнить, что если рассматривать пространство $V C^{1}$ как пространство ядер операторов в подходящих пространствах $L^{p}$, то оно не является инвариантным относительно сопряжения унитарными операторами, в отличие от пространств Шаттена-фон Неймана и, в частности, пространства ядерных операторов. Действительно, в определении $V C^{1}$ существенно участвуют известные сигма-подалгебры, которые не имеют нужной инвариантности. См. работу [2], где рассматривается близкий вопрос. Подробнее о следах ядерных операторов и виртуально непрерывных функциях см. [23].

3.4. Ограничение метрик. Следующая задача была одним из источников проблематики данной работы. Пусть $(X, \mu)$ - стандартное пространство с непрерывной мерой. Предположим, что $\rho$ - допустимая метрика и $\xi-$ измеримое разбиение пространства $(X, \mu)$, элементы которого имеют меру нуль, например, $\xi$ - разбиение на линии уровня функции, не являющейся постоянной ни на каких множествах положительной меры. Можно ли корректно ограничить метрику (как функцию двух переменных) на элементы разбиения?

Ответ на этот вопрос не столь очевиден, так как метрика здесь - априори всего лишь измеримая функция. Однако, как утверждалось выше, всякая допустимая метрика виртуально непрерывна, и поэтому для получения положительного ответа достаточно указать ту бистохастическую меру, на носитель которой следует ограничить метрику. Для простоты будем считать, что $X=[0,1]^{2}, \mu$ - лебегова мера на квадрате, а разбиение $\xi-$ разбиение единичного квадрата на вертикальные отрезки. Тогда речь идет об ограничении виртуально непрерывной функции, заданной на $X^{2}=[0,1]^{4}$, на трехмерное подмногообразие $\left\{\left(x_{1}, x_{2}, x_{3}, x_{4}\right): x_{1}=x_{3}\right\}$. Легко видеть, что это подмногообразие, снабженное трехмерной лебеговой мерой, определяет бистохастическую меру на $X \times X$. 
Авторы признательны Л. Ловасу, приславшему свою недавно вышедшую монографию [7], в которой, в частности, обсуждаются некоторые близкие вопросы, и А. Логунову, обратившему наше внимание на возможность двойственного определения понятия толщины.

\section{Список литературы}

[1] R. A. Adams, J. J.F. Fournier, Sobolev spaces, 2nd ed., Pure Appl. Math. (Amst.), 140, Elsevier/Academic press, Amsterdam, 2003, xiv+305 pp.

[2] M. Denker, M. Gordin, "Limit theorems for von Mises statistics of a measure preserving transformation", Probab. Theory Related Fields, 160:1-2 (2014), 1-45; 2013, 47 pp., arXiv: $1109.0635 \mathrm{v} 2$.

[3] M. Gromov, Metric structure for Riemannian and non-Riemannian spaces, Progr. Math., 152, Birkhäuser Boston, Inc., Boston, MA, 1999, xx+585 pp.

[4] Л.В. Канторович, "О перемещении масс", Докл. АН СССР, 37:7-8 (1942), 227-229; перепеч. в сб.: Теория представлений, динамические системы. ХІ, Специальный выпуск, Зап. науч. сем. ПОМИ, 312, ПОМИ, СПб., 2004, 11-14; англ. пер.: L. V. Kantorovich, "On the translocation of masses", J. Math. Sci. (N. Y.), 133:4 (2006), 1381-1382.

[5] Л.В. Канторович, Г.Ш. Рубинштейн, "Пространство вполне аддитивных функций”, Вестн. Ленингр. ун-та, 13:7 (1958), 52-59.

[6] J. Komlós, "A generalization of a problem of Steinhaus", Acta Math. Acad. Sci. Hungar., 18:1-2 (1967), 217-229.

[7] L. Lovász, Large networks and graph limits, Amer. Math. Soc. Colloq. Publ., 60, Amer. Math. Soc., Providence, RI, 2012, xiv+475 pp.

[8] L. Lovász, Coupling measure concentrated on a given set, $2 \mathrm{pp}$. www.cs.elte.hu/ lovasz/book/homnotes-A-3-4b.pdf.

[9] В. Г. Мазья, Пространства Соболева, Изд-во ЛГУ, Л., 1985, 416 с.; англ. пер.: V. G. Maz'ja, Sobolev spaces, Springer Ser. Soviet Math., Springer-Verlag, Berlin, 1985, xix+486 pp.

[10] A. M. Vershik, P. B. Zatitskiy, F. V. Petrov, "Geometry and dynamics of admissible metrics in measure spaces", Cent. Eur. J. Math., 11:3 (2013), 379-400.

[11] В. А. Рохлин, "Об основных понятиях теории меры", Матем. сб., 25(67):1 (1949), 107-150.

[12] K.-T. Sturm, The space of spaces: curvature bounds and gradient flows on the space of metric measure space, 2012, 75 pp., arXiv: 1208.0434.

[13] А. М. Вершик, "Универсальное пространство Урысона, метрические тройки Громова и случайные метрики на натуральном ряде", УМН, 53:5(323) (1998), 57-64; англ. пер.: А. M. Vershik, "The universal Urysohn space, Gromov metric triples and random metrics on the natural numbers", Russian Math. Surveys, 53:5 (1998), 921-928.

[14] А. М. Вершик, "Классификация измеримых функций нескольких аргументов и инвариантно распределенные случайные матрицы", Функи. анализ и его прил., 36:2 (2002), 12-27; англ. пер.: А. M. Vershik, "Classification of measurable functions of several variables and invariantly distributed random matrices", Funct. Anal. Appl., 36:2 (2002), 93-105.

[15] A. M. Vershik, "Random and universal metric spaces", Dynamics and randomness II, Nonlinear Phenom. Complex Systems, 10, Kluwer Acad. Publ., Dordrecht, 2004, 199-228. 
[16] А. М. Вершик, "Случайные метрические пространства и универсальность", УМН, 59:2(356) (2004), 65-104; англ. пер.: A. M. Vershik, "Random metric spaces and universality", Russian Math. Surveys, 59:2 (2004), 259-295.

[17] A. M. Vershik, "Polymorphisms, Markov processes, and quasi-similarity", Discrete Contin. Dyn. Syst., 13:5 (2005), 1305-1324.

[18] А. М. Вершик, "Как выглядит типичный марковский оператор?", Алгебра и анализ, 17:5 (2005), 91-104; англ. пер.: А. M. Vershik, "What does a generic Markov operator look like?", St. Petersburg Math. J., 17:5 (2006), 763-772.

[19] A. M. Vershik, "Dynamics of metrics in measure spaces and their asymptotic invariants", Markov Process. Related Fields, 16:1 (2010), 169-185.

[20] А. М. Вершик, "О классификации измеримых функций нескольких переменных", Теория представлений, динамические системы, комбинаторные методы. XXI, Зап. науч. сем. ПОМИ, 403, ПОМИ, СПб., 2012, 35-57; англ. пер.: А. M. Vershik, "On classification of measurable functions of several variables", J. Math. Sci. (N. Y.), 190:3 (2013), 427-437.

[21] A. M. Vershik, "Long history of Monge-Kantorovich transportation problem", Math. Intelligencer, 35:4 (2013), 1-9.

[22] А. М. Вершик, П. Б. Затицкий, Ф.В.Петров, "Виртуальная непрерывность измеримых функций многих переменных и теоремы вложения", Функи. анализ и его прил., 47:3 (2013), 1-11; англ. пер.: А. M. Vershik, P. B. Zatitskii, F. V. Petrov, "Virtual continuity of measurable functions of several variables and embedding theorems", Funct. Anal. Appl., 47:3 (2013), 165-173.

[23] А. М. Вершик, П. Б. Затицкий, Ф. В. Петров, "Интегрирование виртуально непрерывных функций по бистохастическим мерам и формула следа ядерных операторов", Алгебра и анализ (в печати).

[24] П. Б. Затицкий, Ф. В. Петров, "Об исправлении метрик", Теория представлений, динамические системь, комбинаторные методы. ХХ, Зап. науч. сем. ПОМИ, 390, ПОМИ, СПб., 2011, 201-209; англ. пер.: P. B. Zatitskiy, F. V. Petrov, "Correction of metrics", J. Math. Sci. (N. Y.), 181:6 (2012), 867-870.

\section{Анатолий Моисеевич Вершик \\ (Anatolii M. Vershik)}

Санкт-Петербургское отделение

Математического института им. В. А. Стеклова РАН;

Санкт-Петербургский государственный университет;

Институт проблем передачи информации

им. А. А. Харкевича РАН

E-mail: avershik@gmail.com

\section{Павел Борисович Затицкий \\ (Pavel B. Zatitskiy)}

Исследовательская лаборатория им. П. Л. Чебышёва при Санкт-Петербургском государственном

университете;

Санкт-Петербургское отделение

Математического института им. В. А. Стеклова РАН

E-mail: paxa239@yandex.ru

\section{Федор Владимирович Петров}

(Fedor V. Petrov)

Санкт-Петербургское отделение

Математического института им. В. А. Стеклова РАН;

Санкт-Петербургский государственный университет

E-mail: fedyapetrov@gmail.com 